\title{
MeerKAT HI commissioning observations of MHONGOOSE galaxy ESO 302-G014
}

\author{
W. J. G. de Blok ${ }^{1,2,3}$, E. Athanassoula ${ }^{4}$, A. Bosma ${ }^{4}$, F. Combes ${ }^{5}$, J. English ${ }^{6}$, G. H. Heald ${ }^{7}$, P. Kamphuis ${ }^{8}$, \\ B. S. Koribalski ${ }^{9}, 10,11$, G. R. Meurer ${ }^{12}$, J. Román ${ }^{13}$, A. Sardone ${ }^{14,15}$, L. Verdes-Montenegro ${ }^{13}$, F. Bigiel ${ }^{16}$,
} E. Brinks ${ }^{17}$, L. Chemin ${ }^{18}$, F. Fraternali ${ }^{3}$, T. Jarrett ${ }^{2}$, D. Kleiner ${ }^{19}$, F. M. Maccagni ${ }^{19}$, D. J. Pisano ${ }^{20,21,22}$, P. Serra ${ }^{19}$, K. Spekkens ${ }^{23}$, P. Amram ${ }^{4}$, C. Carignan ${ }^{24,2}$, R.-J. Dettmar ${ }^{8}$, B. K. Gibson ${ }^{25}$, B. W. Holwerda ${ }^{26}$, G. I. G. Józsa ${ }^{27,28,16}$, D. M. Lucero ${ }^{29}$, T. A. Oosterloo ${ }^{1,3}$, A. J. T. Ramaila ${ }^{27}$, M. Ramatsoku ${ }^{28,19}$, K. Sheth ${ }^{30}$, F. Walter ${ }^{31}$, O. I. Wong ${ }^{7,12,11}$, A. A. Zijlstra ${ }^{32}$, S. Bloemen ${ }^{33}$, P. J. Groot ${ }^{33,34,35}$, R. Le Poole ${ }^{36}$, M. Klein-Wolt ${ }^{33}$, E. G. Körding ${ }^{33}$, V. A. McBride ${ }^{37}$, K. Paterson ${ }^{38}$, D. L. A. Pieterse ${ }^{33}$, P. Vreeswijk ${ }^{33}$, and P. A. Woudt ${ }^{34}$

(Affiliations can be found after the references)

Received 11 July 2020 / Accepted 9 September 2020

\begin{abstract}
Aims. We present the results of three commissioning H I observations obtained with the MeerKAT radio telescope. These observations make up part of the preparation for the forthcoming MHONGOOSE nearby galaxy survey, which is a MeerKAT large survey project that will study the accretion of gas in galaxies and the link between gas and star formation.

Methods. We used the available H I data sets, along with ancillary data at other wavelengths, to study the morphology of the MHONGOOSE sample galaxy, ESO 302-G014, which is a nearby gas-rich dwarf galaxy.

Results. We find that ESO 302-G014 has a lopsided, asymmetric outer disc with a low column density. In addition, we find a tail or filament of H I clouds extending away from the galaxy, as well as an isolated H I cloud some $20 \mathrm{kpc}$ to the south of the galaxy. We suggest that these features indicate a minor interaction with a low-mass galaxy. Optical imaging shows a possible dwarf galaxy near the tail, but based on the current data, we cannot confirm any association with ESO 302-G014. Nonetheless, an interaction scenario with some kind of low-mass companion is still supported by the presence of a significant amount of molecular gas, which is almost equal to the stellar mass, and a number of prominent stellar clusters, which suggest recently triggered star formation.

Conclusions. These data show that MeerKAT produces exquisite imaging data. The forthcoming full-depth survey observations of ESO $302-\mathrm{G} 014$ and other sample galaxies will, therefore, offer insights into the fate of neutral gas as it moves from the intergalactic medium onto galaxies.
\end{abstract}

Key words. galaxies: clusters: individual: ESO 302-G014 - galaxies: dwarf - galaxies: ISM - radio lines: galaxies

\section{Introduction}

The relation between the gas content of a disc galaxy and the magnitude and distribution of star formation is an important topic in the study of galaxy evolution. We can split this topic up into two components.

The first one pertains to the detailed physical conditions for in situ disc gas to turn into stars. Much of this focuses on the conversion from molecular gas to stars, but the atomic to molecular hydrogen conversion is another key step in this process. The global relation between gas density (atomic neutral hydrogen, or $\mathrm{HI}$, and molecular gas) and star-formation rate (SFR), also known as the Kennicutt-Schmidt law (Schmidt 1959; Kennicutt 1998), is well-characterised. Comprehensive and detailed studies of the sub-kpc relations have also become available in recent years. Some examples include studies based on The H I Nearby Galaxy Survey (THINGS, Walter et al. 2008) and its data (Bigiel et al. 2008; Leroy et al. 2008). Other recent works include Zheng et al. (2013) on the regulation of star formation; as well as Thilker et al. (2007) and Holwerda et al. (2012) on the link between star formation and H I morphology (implying a physical link; Heiner et al. 2008); studies of multiscale star formation and H I (Elson et al. 2019); the link between SFR and gas based on jellyfish galaxies (Jáchym et al. 2019;
Ramatsoku et al. 2019, 2020; Moretti et al. 2020; Bacchini et al. 2019) on the link between SFR and H I volume densities.

The second component deals with the origin of the neutral hydrogen that turns into stars. In many local disc galaxies the gas depletion time scale, which is the time scale after which galaxies run out of molecular gas (at their current SFRs), is only a few Gyr (Bigiel et al. 2011). The molecular gas reservoir can be replenished from the neutral hydrogen present in these galaxies, but we do not observe the corresponding decline that is expected in the amount of cold neutral gas in galaxies over cosmic time (e.g. Noterdaeme et al. 2012; Kanekar et al. 2016; Hu et al. 2019; Sardone et al., in prep.).

The conventional explanation is that galaxies acquire more gas over their lifetime by accretion from the intergalactic medium (IGM). However, in an inventory of possible H I accretion features in the literature data available at the time, Sancisi et al. (2008) found that H I accretion can account for only 20\% of the gas supply needed to sustain the current SFR in galaxies. Most of the observations from the literature used for their study had H I column density limits of $\sim 10^{20} \mathrm{~cm}^{-2}$. A natural explanation of this discrepancy is that accretion still occurs at lower column densities, possibly in the form of partly ionised gas filaments. The latter process has become known as "cold accretion" (Kereš et al. 2005; Nelson et al. 2013, 2015; Schaller et al. 2015). Recent 
simulations highlight the importance of accretion for galaxy evolution (van de Voort et al. 2019).

The Westerbork Hydrogen Accretion in LOcal GAlaxieS (HALOGAS) survey (Heald et al. 2011) was one of the first systematic attempts to detect this $\mathrm{HI}$ accretion and to put observational limits on the $\mathrm{HI}$ accretion rate at lower column densities $\left(\sim 10^{19} \mathrm{~cm}^{-2}\right)$ in a sample of 24 gas-rich disc galaxies. However, even with these more sensitive data, the resulting $\mathrm{H} \mathrm{I}$ accretion rate is still significantly lower than the observed SFR (Kamphuis et al., in prep.). Most of the low-column density gas that is detected in HALOGAS is extra-planar disc gas that most likely originates in the galactic fountain process (Marasco et al. 2019 and references therein; also see Lucero et al. 2015).

Several possible explanations have been proposed for this lack of visible accretion. The first is that the external accretion process can only, or mainly, be observed at low column densities well below $10^{19} \mathrm{~cm}^{-2}$. Simulations indicate that the distribution of $\mathrm{HI}$ should be much more extended at column densities around $\sim 10^{17-18} \mathrm{~cm}^{-2}$ than at $10^{19-20} \mathrm{~cm}^{-2}$ (e.g. Popping et al. 2009; van de Voort et al. 2019) and some of this low column density H I could make up the accreting material. Ionised gas at relatively low column densities is observed around star-forming galaxies (e.g. Tumlinson et al. 2017; Werk et al. 2013, 2014) but its properties and origin are difficult to establish from absorption measurements alone and, ideally, it should be seen in emission.

A second explanation - which does not rule out the first is that direct cold accretion is not the dominant mechanism, but that accretion happens through clouds that are launched from the disc as part of the galactic fountain process. These clouds are launched from the disc into the hot halo, where they accrete gas which cools in their wake. This gas is then brought back to the disc (Fraternali et al. 2013; Fraternali 2017).

Whatever the mechanism, the low-column density material is most likely to be clumpy on smaller $(\sim \mathrm{kpc})$ scales (Popping et al. 2009; Wolfe et al. 2016), so for the next observational step, both good column density sensitivity and spatial resolution are needed. The MHONGOOSE survey (MeerKAT H I Observations of Nearby Galactic Objects: Observing Southern Emitters; de Blok et al. 2016) with the MeerKAT telescope will have both of these. It will probe the resolved distribution of $\mathrm{H}$ I at uniquely deep column densities, allowing us to reveal what is possibly accreting cold gas and quantify its contribution compared to the recycled gas from the fountain process, thereby further opening up the observational parameter space to probe the accretion of gas in galaxies.

The MeerKAT radio synthesis telescope (Jonas \& MeerKAT Team 2016; Camilo 2018; Mauch et al. 2020) is a 64-dish interferometer located in the Karoo desert in South Africa. Each dish has a Gregorian design with an effective $13.5 \mathrm{~m}$ diameter. MeerKAT is a precursor telescope of the future SKA1-MID telescope (Braun et al. 2015). It will eventually also form part of this telescope.

Much of the MeerKAT science case is addressed in a number of Large Survey Projects (LSPs) which are multi-year observing projects with $>1000 \mathrm{~h}$ of observing time apiece. MHONGOOSE (de Blok et al. 2016) is an LSP specifically designed to provide both high-resolution and high-sensitivity observations of nearby galaxies, which is handy in addressing the two main components of the link between gas content and star formation described above.

A full description of the MHONGOOSE survey, the sample, and the sample selection will be given in a future paper. Here, we give a brief summary of the essentials only. MHONGOOSE will observe 30 disc and dwarf galaxies over a total of $1650 \mathrm{~h}(55 \mathrm{~h}$ per galaxy) over the next five years. The sample covers five bins in logarithmic $\mathrm{H}$ I mass in the range $6<\log \left(M_{\mathrm{HI}} / M_{\odot}\right)<11$ with an equal number of galaxies in each bin. These galaxies were selected from the Survey for Ionization in Neutral Gas Galaxies (SINGG) (Meurer et al. 2006) sample. SINGG's main goal is to provide a census of star formation in $\mathrm{H} \mathrm{I}$-selected galaxies using $\mathrm{H} \alpha$ and $R$-band observations. Galaxies with specific inclinations and SFRs were selected to optimise the modelling and analysis and to ensure a comprehensive coverage of the H I mass and SFR distribution. For completeness, we list the sample and some basic properties of these galaxies in Table A.1, but, as noted, a full description will be given in a future paper.

The expected H I column density sensitivity of the MHONGOOSE observations is $\sim 3 \times 10^{18} \mathrm{~cm}^{-2}$ at $3 \sigma$ over a $16 \mathrm{~km} \mathrm{~s}^{-1}$ channel at $30^{\prime \prime}$ resolution. This implies that at $\sim 1^{\prime}$ resolution the column density sensitivity will be equal to those of the deepest Parkes or Green Bank telescope (GBT) H I observations of the MHONGOOSE galaxies (Sorgho et al. 2019; Sardone et al., in prep.), but at an angular resolution an order of magnitude finer (cf. Fig. 5 in de Blok et al. 2016).

Similarly at $\sim 7^{\prime \prime}$, the highest resolution we can achieve for $\mathrm{HI}$ in MHONGOOSE, the column density sensitivity will be a factor of four better $\left(\sim 6 \times 10^{19} \mathrm{~cm}^{-2}\right.$ at $3 \sigma$ over a $16 \mathrm{~km} \mathrm{~s}^{-1}$ channel at $7^{\prime \prime}$ ) than the THINGS observations (Walter et al. 2008), enabling the mapping of $\mathrm{H}$ I beyond the star-forming disc at these very high resolutions.

The spectral resolution of the final observations will be $3.3 \mathrm{kHz}$, or $0.7 \mathrm{~km} \mathrm{~s}^{-1}$ at the $\mathrm{H}$ I rest frequency of $1420.4 \mathrm{MHz}$, using the narrow-band mode of the MeerKAT correlator, dividing the bandwidth of $107 \mathrm{MHz}$ available in that mode into 32768 channels. This will enable highly accurate observations of the H I profiles, with the ability to separate them into cold and warm components, thus tracing the different neutral hydrogen components of the interstellar medium (ISM).

As part of the MeerKAT telescope commissioning, one of the MHONGOOSE galaxies, ESO 302-G014, was observed multiple times over the course of two years at various stages of completeness of the array and the correlator. The initial choice for this galaxy was its observability at night during the first observations and the lack of high-quality resolved H I data in telescope archives at the time.

In this paper, we describe three MeerKAT commissioning H I data sets from ESO 302-G014. We also present a brief discussion of the morphology of the galaxy and possible scenarios for its evolution. Section 2 describes the properties of the galaxy. Section 3 gives details on the observations and data reduction. Section 4 describes the data and presents a first analysis. Section 5 presents a short discussion. We summarise our work in Sect. 6, including our expectations for the full survey. Unless noted otherwise, all velocities mentioned in this paper are heliocentric.

\section{ESO 302-G014}

ESO 302-G014 (HIPASS J0351-38) is a low-mass, late-type and gas-rich dwarf galaxy. The SINGG $R$-band image of ESO 302G014 is shown in Fig. 1. The galaxy has an absolute magnitude of $M_{B}=-15.33$ (Prieto et al. 2012), making it somewhat less luminous than the SMC. As projected on the sky, it is located between the Fornax cluster and the Dorado group (Venhola et al. 2018), but it is not itself part of any group (Kourkchi \& Tully 2017). The galaxy has a recessional velocity of $871 \mathrm{~km} \mathrm{~s}^{-1}$, translating into an assumed distance of $11.7 \mathrm{Mpc}$, as derived in Meurer et al. (2006) from the heliocentric velocity using the 
model by Mould et al. (2000). At this distance, $1^{\prime \prime}$ corresponds to $57 \mathrm{pc}$ and $1^{\prime}$ to $3.4 \mathrm{kpc}$.

It is the host galaxy of SN2008jb, a core-collapse Type II supernova extensively studied by Prieto et al. (2012). They find that the supernova occurred in an actively star-forming part of the galaxy, dominated by two young stellar clusters (visible as the two brightest sources $\sim 45^{\prime \prime}$ to the east of the center of the galaxy in Fig. 1). Various estimates lead them to conclude that the time scale of the star formation in this area is $\sim 9 \mathrm{Myr}$, which would imply a high-mass progenitor for the supernova.

The star-formation rate based on SINGG H $\alpha$ data (Meurer et al. 2006) (for an assumed initial mass function with a Salpeter 1955 slope and spanning the mass range 0.1 to $100 M_{\odot}$ ) is $\sim 0.03 M_{\odot} \mathrm{yr}^{-1}$ (G. Meurer, priv. comm.). This agrees reasonably well with the SFR derived from ultra-violet GALEX data (Meurer et al. 2009; Lee et al. 2011).

These numbers, combined with a stellar mass estimate of $4 \times 10^{7} M_{\odot}$ (Prieto et al. 2012) and an H I mass of $3 \times 10^{8} M_{\odot}$ (Meurer et al. 2006, the H I mass will be discussed extensively in the current paper) lead to SFR values per unit stellar and $\mathrm{HI}$ mass of $\log \left(\mathrm{SFR} / M_{\star}\right) \simeq-9.1\left[\mathrm{yr}^{-1}\right]$ and $\log \left(\mathrm{SFR} / M_{\mathrm{HI}}\right) \simeq-9.9$ $\left[\mathrm{yr}^{-1}\right]$, which are typical for local star-forming dwarf galaxies (cf. Lee et al. 2011).

An independent stellar mass determination using WISE data confirms this picture. Based on the $\mathrm{W} 1$ flux and the relations from Cluver et al. (2014), we find a value of $\log \left(M_{\star} / M_{\odot}\right)=$ $7.82 \pm 1.82$ or $M_{\star} \sim 6.6 \times 10^{7} M_{\odot}$. The Spitzer $\mathrm{S} 4 \mathrm{G}$ data report a stellar mass of $1.1 \times 10^{8} M_{\odot}$, corrected to the distance adopted here; compare with Muñoz-Mateos et al. (2015).

These numbers, again, lead to a specific SFR that is normal for dwarf galaxies. They do show, however, that the galaxy is very gas rich. Depending on the stellar mass value adopted, the mass ratio $M_{\mathrm{HI}} / M_{\star}$ varies between $\sim 2.9$ and $\sim 7.5$.

The occurrence of SN2008jb and the relative (projected) proximity to the Fornax cluster has led to the availability of much archival data for this galaxy, including observations by ALMA and HST. As these data sets are used later in this paper, we briefly list their technical details here.

The ALMA $12 \mathrm{~m}$ total power (TP) array observations were carried out as part of a programme to observe 64 Fornax galaxies in band 3, during cycle 5 as part of the project 2017.1.00129.S (PI K. Morokuma). For ESO 302-G014, J0406-3826 was observed as a calibrator. The $\operatorname{CO}(J=1-0)$ emission line was included in the highest frequency USB spectral window (SPW17), while the three others served as continuum. The TP on-the-fly observations were carried out for $2^{\mathrm{h}} 45^{\mathrm{m}}$ in May 2018 with three $12 \mathrm{~m}$-antennae. The angular resolution of the TP observations is $54^{\prime \prime}$, and the velocity resolution $2.9 \mathrm{~km} \mathrm{~s}^{-1}$. The data were calibrated with the Common Astronomy Software Applications (CASA, McMullin et al. 2007). A data cube was produced spanning $212^{\prime \prime} \times 212^{\prime \prime}$, and $4800 \mathrm{~km} \mathrm{~s}^{-1}$. The noise level is $0.16 \mathrm{Jy} \mathrm{beam}^{-1}$ in channels of $10 \mathrm{~km} \mathrm{~s}^{-1}$. The emission is marginally resolved and an integrated $\mathrm{CO}$ spectrum was produced by integrating over an area of $130^{\prime \prime} \times 130^{\prime \prime}$ centered on the optical position.

The HST data consist of a ACS/WFC3 pointing in four filters $(F 606 W, F 336 W, F 657 N, F 160 W)$ obtained as follow-up of SN2008jb as part of Proposal 12992 (P.I. J. Prieto) in October 2012. Reduced data were retrieved from the Hubble Legacy Archive.

In addition, we used data from the Dark Energy Camera Legacy Survey (DECaLS; Dey et al. 2019). The $g, r$, and $z$ images have surface brightness limits of respectively 29.2, 29.0, and $27.5 \mathrm{mag} \operatorname{arcsec}^{-2}\left(3 \sigma\right.$ in $10^{\prime \prime} \times 10^{\prime \prime}$ boxes $)$ in the area of

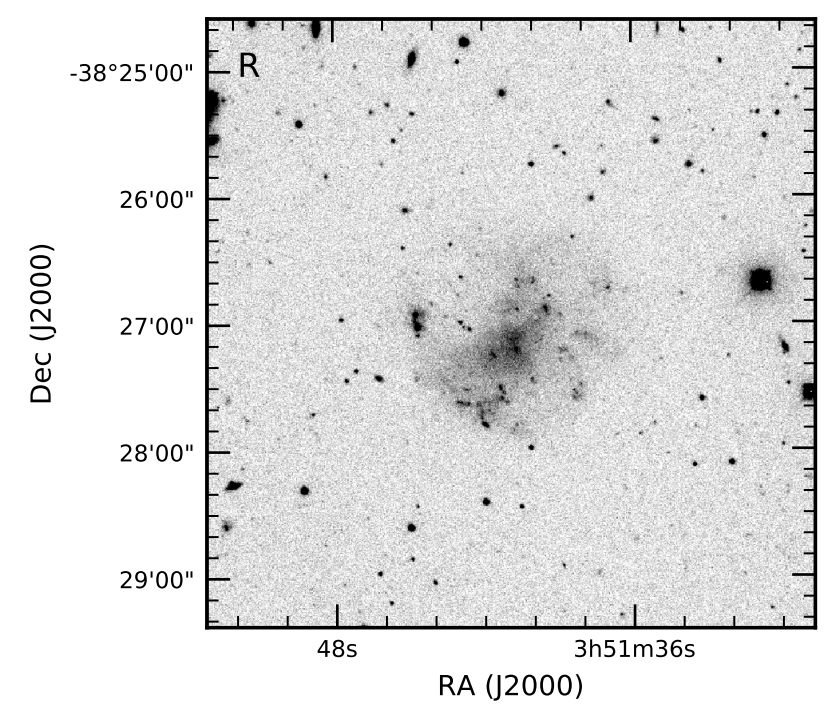

Fig. 1. SINGG $R$-band image of ESO 302-G014 (Meurer et al. 2006). The star clusters discussed in the text are visible as the two brightest sources $45^{\prime \prime}$ east of the center of the galaxy. The limiting surface brightness of this image is $27.6 \mathrm{AB}$ mag $\operatorname{arcsec}^{-2}$.

ESO 302-G014. These ancillary data sets are all discussed in Sect. 5.2.

\section{MeerKAT observations and data reduction}

The first commissioning observations of ESO 302-G014 were done in early 2018 with the MeerKAT-16 array. This early commissioning array consisted of 16 MeerKAT dishes with short baselines. These limited-resolution data showed interesting features in this galaxy (described below), compelling us to target it in subsequent commissioning stages with the full MeerKAT64 array as described in this paper. These further observations consisted of an observation in 2019 using the final MeerKAT SKARAB correlator in its $4 \mathrm{k}$ mode (channel width of $44 \mathrm{~km} \mathrm{~s}^{-1}$ ), and an observation in early 2020 with the full array using the $32 \mathrm{k}$ mode (channel width of $5.5 \mathrm{~km} \mathrm{~s}^{-1}$ ). The final MHONGOOSE observations will have a velocity resolution that is another factor of eight better, giving a channel width of $0.7 \mathrm{~km} \mathrm{~s}^{-1}$. The data described below were not Hanning-smoothed.

\subsection{MeerKAT-16 observation}

ESO 302-G014 was observed in January 2018 with the MeerKAT-16 array. The standard calibrator B1934-638 was used as the flux calibrator, J0408-6548 was used as the bandpass calibrator, and B0451-282 as the gain calibrator. The flux and bandpass calibrators were observed for five minutes each every two hours, with the gain calibrator observed for two minutes every ten minutes. The (now decommissioned) ROACH2 correlator was used, with a channel width of $26.1 \mathrm{kHz}\left(5.5 \mathrm{~km} \mathrm{~s}^{-1}\right.$ at $1420.4 \mathrm{MHz}$ ). Further details of the observation are given in Table 1.

The data were reduced using a pre-release version of the Containerized Automated Radio Astronomy Calibration (CARACal) pipeline (Józsa et al. 2020; see also Serra et al. 2019). This is a containerised pipeline which allows for the automatic running of the relevant components from different radio astronomy packages as part of one single scripted execution. The usual steps of flagging data, cross-calibration, splitting of data, 
Table 1. Observation details.

\begin{tabular}{|c|c|c|c|}
\hline & MeerKAT-16 32k & MeerKAT-64 4k & MeerKAT-64 32k \\
\hline Observing date (UTC) & 28 Jan 2018 & 21 July 2019 & 07 Jan 2020 \\
\hline Observing time (UTC) & $12^{\mathrm{h}} 03^{\mathrm{m}}-23^{\mathrm{h}} 23^{\mathrm{m}}$ & $01^{\mathrm{h}} 06^{\mathrm{m}}-12^{\mathrm{h}} 47^{\mathrm{m}}$ & $15^{\mathrm{h}} 40^{\mathrm{m}}-21^{\mathrm{h}} 53^{\mathrm{m}}$ \\
\hline Array configuration & 16 dishes $^{(a)}$ & 61 dishes $^{(b)}$ & 59 dishes $(c)$ \\
\hline Total observing time & $680 \mathrm{~min}$ & $620 \mathrm{~min}$ & $373 \mathrm{~min}$ \\
\hline Total time on target & $504 \mathrm{~min}$ & $340 \mathrm{~min}$ & $258 \mathrm{~min}$ \\
\hline Channel width & $26.1 \mathrm{kHz}$ & $208.9 \mathrm{kHz}$ & $26.1 \mathrm{kHz}$ \\
\hline Number of channels & 32768 & 4096 & 32768 \\
\hline
\end{tabular}

Notes. ${ }^{(a)}$ Antennas m000, m006, m011, m012, m015, m016, m021, m023, m024, m027, m029, m031, m033, m034, m035, m039. ${ }^{(b)}$ Does not include antennas m001, m025, m039. ${ }^{(c)}$ Does not include antennas m026, m028, m032, m051, m063.

self-calibration, continuum subtraction, as well as line imaging and deconvolution can all be done with a single call to the pipeline. Furthermore, the pipeline provides diagnostic plots that can be used to assess the quality of the data and the calibration and reduction. Especially in the early stages of the reduction, the pipeline can be run in stages and on subsets of the data so that diagnostic plots and output products can be inspected and if necessary, pipeline parameters can be adjusted before a full run on the complete data set.

Using the pipeline, continuum was subtracted in two stages. Firstly, the final self-calibration continuum model was subtracted and, afterwards, any remaining residual continuum was subtracted in the $u v$-plane using a first-order polynomial fit to adjacent line-free channels.

The pipeline then produced the final data cube using a twostep deconvolution process where the output of a first, shallow deconvolution run was used as a mask for the second, deeper deconvolution run. Further details on this data set are given in Table 2. We refer to this observation as the "MeerKAT-16" data.

\subsection{MeerKAT-64 4k observation}

ESO 302-G014 was observed again in July 2019 with the full MeerKAT array, with 61 dishes participating in the observation. The $L$-band receivers were used, covering a frequency range from approximately $900-1670 \mathrm{MHz}$. The SKARAB correlator in $4 \mathrm{k}$ mode was used, dividing the band into 4096 channels with a channel width of $208.9 \mathrm{kHz}$ each, or $\sim 44 \mathrm{~km} \mathrm{~s}^{-1}$. Observations were taken in full polarization mode.

The standard calibrator J0408-6544 was observed as the flux and bandpass calibrator, with J0440-4333 as the gain calibrator. The target and calibrators were observed sequentially, in a cycle of $10 \mathrm{~min}$ on target, $5 \mathrm{~min}$ on J0408-6544, and 2 min on J04404333. The total time spent on the flux and bandpass calibrator is longer than usual, but this allowed for an accurate study of the various calibration steps for commissioning purposes. Further details of the observation are listed in Table 1.

The data were again reduced using a pre-release version of the CARACal pipeline. After delay, bandpass and gain calibration solutions were derived, three rounds of self-calibration were then applied to the data. A continuum image was created using the central 100 channels $(20.9 \mathrm{MHz}$ centered on $1416.5 \mathrm{MHz})$, using a Briggs robustness weighting parameter (Briggs 1995) of $r=0.0$. The noise in this image is $14 \mu \mathrm{Jy}$ with a beam size of $8.2^{\prime \prime} \times 7.1^{\prime \prime}$. The most prominent feature in the continuum image at this angular resolution is a peak at the location of SN2008jb. We find a peak brightness at that location of $96.8 \mu \mathrm{Jy} \mathrm{beam}^{-1}$. The continuum image is discussed further in Sect. 5.3.
The continuum emission was removed using the source list derived from the clean model of the final self-calibrated continuum image followed by a linear fit using the channels straddling the H I emission, and a subsequent subtraction in the $u v$ plane. We extracted the central eight channels that were then deconvolved in a two-step process as described above.

We produced a number of data cubes this way, using various values of the robust parameter $r$ to explore the image quality and the H I morphology at various resolutions. Table 2 lists the resulting synthesised beam values, as well as noise levels and column density limits. The latter is calculated as a $1 \sigma$ limit for a single $209 \mathrm{kHz}$ channel. In addition, we also show the $3 \sigma$ column density limits for a fixed line width to facilitate direct comparison with the other two data sets.

The natural-weighted data set has a noise per channel of $85 \mu \mathrm{Jy}_{\mathrm{beam}^{-1}}$. We calculate an expected thermal noise of $79 \mu \mathrm{Jy}_{\text {beam }}{ }^{-1}$, showing that the noise levels in the data are close to the expected value. In the rest of this paper, we refer to this dataset as the " $4 \mathrm{k}$ " data.

\subsection{MeerKAT-64 32k observation}

Our final ESO 302-G014 commissioning observation took place in January 2020, this time as part of commissioning of the $32 \mathrm{k}$ mode of the SKARAB correlator. This covers a total bandwidth of $856 \mathrm{MHz}$ with 32768 channels that are each $26.1 \mathrm{kHz}$ wide. This corresponds to $5.5 \mathrm{~km} \mathrm{~s}^{-1}$ at $1.4 \mathrm{GHz}$. The full array was used, with 59 active dishes.

The standard calibrator J0408-6544 was observed as the primary flux and bandpass calibrator, J0440-4333 as the gain calibrator. The target and phase calibrator were observed sequentially, in a cycle of $10 \mathrm{~min}$ on target, and $2 \mathrm{~min}$ on the secondary calibrator. The primary calibrator was observed twice during the observation for a total of $20 \mathrm{~min}$. Additional observing parameters are listed in Table 1.

The reduction of the data was identical to that described above, except that (in order to speed up the reduction process) we ran the self-calibration step on a smaller measurement set created by binning the data in bins of 40 channels wide. The resulting self-calibration solutions were then interpolated and applied to the measurement set with the original spectral resolution. Continuum subtraction and creation and deconvolution of data cubes then proceeded as above.

The cube produced with a robust value $r=1.0$ (close to natural-weighted) has a noise per channel of $255 \mu \mathrm{Jy} \mathrm{beam}^{-1}$. The expected thermal noise is $226 \mu \mathrm{Jy}_{\text {beam }}{ }^{-1}$, again showing good agreement. In the rest of this paper, we refer to this dataset as the " $32 \mathrm{k}$ " data. 
Table 2. Noise levels for the H I cubes.

\begin{tabular}{|c|c|c|c|c|c|c|c|c|c|}
\hline Array & Corr. & $r$ & Noise $\sigma$ & Beam & PA (beam) & $N_{\mathrm{HI}}$ & $N_{\mathrm{HI}}^{3 \sigma}$ & \multirow{3}{*}{$\begin{array}{l}F \\
\left(\mathrm{Jy} \mathrm{km} \mathrm{s}^{-1}\right) \\
(9)\end{array}$} & \multirow{3}{*}{$\begin{array}{l}M_{\mathrm{HI}} \\
\left(10^{8} M_{\odot}\right) \\
(10)\end{array}$} \\
\hline & mode & & $\left(\mu \mathrm{Jy}_{\text {beam }}{ }^{-1}\right)$ & $(\operatorname{arcsec})$ & $\left({ }^{\circ}\right)$ & \multicolumn{2}{|c|}{$\left(10^{18} \mathrm{~cm}^{-2}\right)$} & & \\
\hline (1) & (2) & (3) & (4) & (5) & (6) & $(7)$ & (8) & & \\
\hline MeerKAT-16 & $32 \mathrm{k}$ & 2.0 & 1040 & $62.7 \times 54.2$ & 68.4 & 1.9 & 9.7 & $10.93 \pm 0.29$ & $3.5 \pm 0.1$ \\
\hline \multirow[t]{4}{*}{ MeerKAT-64 } & $4 \mathrm{k}$ & 0.0 & 120 & $8.0 \times 7.5$ & 97.5 & 97.8 & $58.7^{(a)}$ & 10.70 & 3.5 \\
\hline & $4 \mathrm{k}$ & 0.5 & 97 & $11.5 \times 10.1$ & 113.2 & 40.8 & $24.5^{(a)}$ & 11.69 & 3.8 \\
\hline & $4 \mathrm{k}$ & 1.0 & 86 & $23.7 \times 16.6$ & 124.6 & 10.7 & $6.4^{(a)}$ & 12.14 & 3.9 \\
\hline & $4 \mathrm{k}$ & 1.5 & 85 & $30.7 \times 21.8$ & 122.9 & 6.2 & $3.7^{(a)}$ & 11.93 & 3.9 \\
\hline \multirow[t]{4}{*}{ MeerKAT-64 } & $32 \mathrm{k}$ & 0.0 & 358 & $8.2 \times 6.8$ & 155.5 & 39.3 & 201.2 & $10.45 \pm 0.16$ & $3.4 \pm 0.1$ \\
\hline & $32 \mathrm{k}$ & 0.5 & 283 & $13.7 \times 9.8$ & 139.7 & 12.9 & 66.0 & $11.28 \pm 0.14$ & $3.6 \pm 0.1$ \\
\hline & $32 \mathrm{k}$ & 1.0 & 255 & $25.4 \times 18.7$ & 139.4 & 3.3 & 16.9 & $11.98 \pm 0.11$ & $3.9 \pm 0.1$ \\
\hline & $32 \mathrm{k}$ & 1.5 & 247 & $30.3 \times 25.9$ & 143.1 & 1.9 & 9.7 & $11.87 \pm 0.09$ & $3.8 \pm 0.1$ \\
\hline
\end{tabular}

Notes. ${ }^{(a)}$ These column densities are scaled from values measured with a wider $45 \mathrm{~km} \mathrm{~s}^{-1}$ channel and are listed here merely for comparison. They cannot be measured directly from the data set. (1): MeerKAT array configuration. (2) Correlator mode. The 4k mode has a channel width of $44 \mathrm{~km} \mathrm{~s}^{-1}$; the $32 \mathrm{k}$ mode $5.5 \mathrm{~km} \mathrm{~s}^{-1}$. (3) Value of the robustness parameter $r$. (4) Noise $\sigma$ in a single channel in $\mu \mathrm{Jy}_{\text {beam }}{ }^{-1}$. (5) Size of the synthesised beam in arcseconds. (6) Position angle of the major axis of the beam. (7) The $1 \sigma$ column density sensitivity in a single channel $\left(\times 10^{18} \mathrm{~cm}^{-2}\right)$. (8) The $3 \sigma$ column density for a $16 \mathrm{~km} \mathrm{~s}^{-1}$ line width (assuming a single channel). (9) Integrated H I flux $F$ (in Jy km s$)^{-1}$ ) with associated uncertainty. No uncertainty is given for the $4 \mathrm{k}$ data, due to the small number of channels with emission. (10) Total H I mass, derived from Col. (9) and assuming a distance of $11.7 \mathrm{Mpc}\left(\times 10^{8} M_{\odot}\right)$.

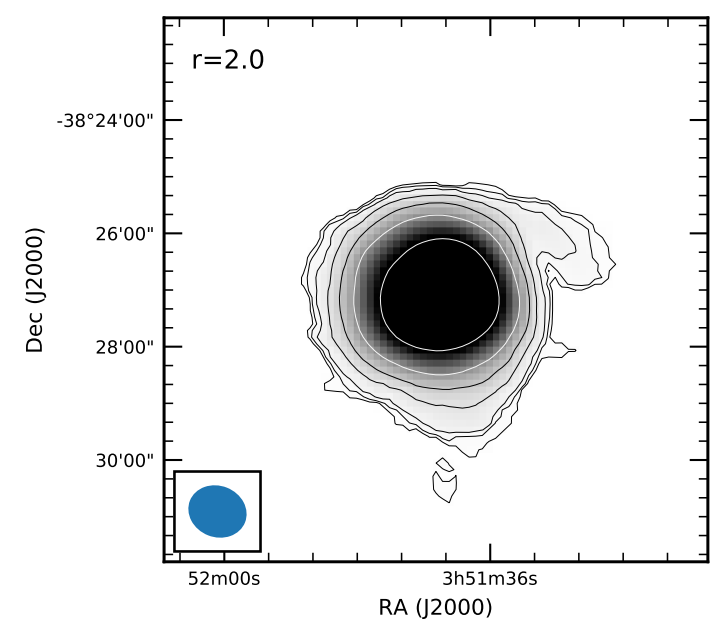

Fig. 2. Integrated H I map (zeroth moment) of the MeerKAT-16 data using $r=2.0$. Contour levels are $(0.05,0.1,0.2,0.5,1.0,2.0,5.0$, $10.0) \times 10^{20} \mathrm{~cm}^{-2}$. The MeerKAT-16 beam is shown in the bottom-left corner. We note the extension visible in the north-western part of the disc.

\section{Description of the HI data}

In Table 2, we list the noise levels per channel for the MeerKAT16 as well as the MeerKAT-64 4k and 32k data, using different robust values $r$. Also listed are the resulting beam sizes and $1 \sigma$ single-channel column-density sensitivities.

For the MeerKAT-16 data, we do not show the channel maps due to the limited angular resolution of the data, which, of course, is massively improved by the MeerKAT-64 data. Instead, we show in Fig. 2 the integrated H I or zeroth-moment map, as this was the motivation to use this galaxy for further commissioning observations.

The map was created using the SoFiA source finder (Serra et al. 2015). We searched for emission brighter than $3 \sigma$ using all four combinations of the original spatial and velocity resolution, a Gaussian spatial smoothing kernel equal to the synthesised beam, and a three-channel boxcar velocity smoothing kernel. The built-in comparison between positive and negative detections with a reliability threshold of 0.90 was then used to distinguish between noise peaks and real emission.

In Fig. 2, despite the limited resolution, we clearly see a filament-like structure emanating from the northern part of the disc and extending to the west-south-west. This intriguing feature led us to use ESO 302-G014 for future commissioning observations as well. We discuss the feature in more detail below.

In Fig. 3, we present the three channel maps containing $\mathrm{H}_{\mathrm{I}}$ emission from the $4 \mathrm{k}$ data created using a robustness value of $r=$ 0.5. Most of the emission is concentrated in the central channel, which is consistent with the velocity width of the galaxy and the large channel width. The central velocity channel clearly shows the extension to the west, along with some possible faint clouds at $\sim 7.5^{\prime}$ south (around $\left.(\alpha, \delta)=3^{\mathrm{h}} 51^{\mathrm{m}} 24^{\mathrm{s}},-38^{\circ} 35^{\prime} 30^{\prime \prime}\right)$.

The same velocity range is shown in Fig. 4 for the $32 \mathrm{k}$ data (taking into account the very different channel widths). Here. we show only every second channel.

A comparison with the $4 \mathrm{k}$ channel maps in Fig. 3 shows a marked increase in the amount of detail in the $32 \mathrm{k}$ data. A comparison is useful, as many wideband continuum studies with MeerKAT will use the $4 \mathrm{k}$ correlator mode. The high sensitivity of MeerKAT will allow for the detection and characterisation of $\mathrm{H} \mathrm{I}$ in galaxies over a large fraction of the band, despite the wide channels in the $4 \mathrm{k}$ mode. Knowledge of how the observed characteristics of this $\mathrm{H}$ I can differ between $4 \mathrm{k}$ and $32 \mathrm{k}$ data will thus contribute to a better understanding of the limitations of these $4 \mathrm{k}$ H I data.

The channel maps in Fig. 4 show details in the tail (indicated in the $892.5 \mathrm{~km} \mathrm{~s}^{-1}$ channel map) and, together with Figs. 2 and 3, establish that it winds clockwise. There are also two features in the south. The one in the south-east of the main body of the galaxy becomes visible starting from the channel at $837.4 \mathrm{~km} \mathrm{~s}^{-1}$ (marked " 1 " in that channel) and persists in the two subsequent channels. Its winding is anti-clockwise, that is, opposite to that of the main feature in the north. There is also a second feature in the south which becomes clearly visible starting from the channel at $848.4 \mathrm{~km} \mathrm{~s}^{-1}$ (marked " 2 " in that channel) and also persists in a couple of subsequent channels. Its winding is clockwise, that is, the same as the main northern feature. This 


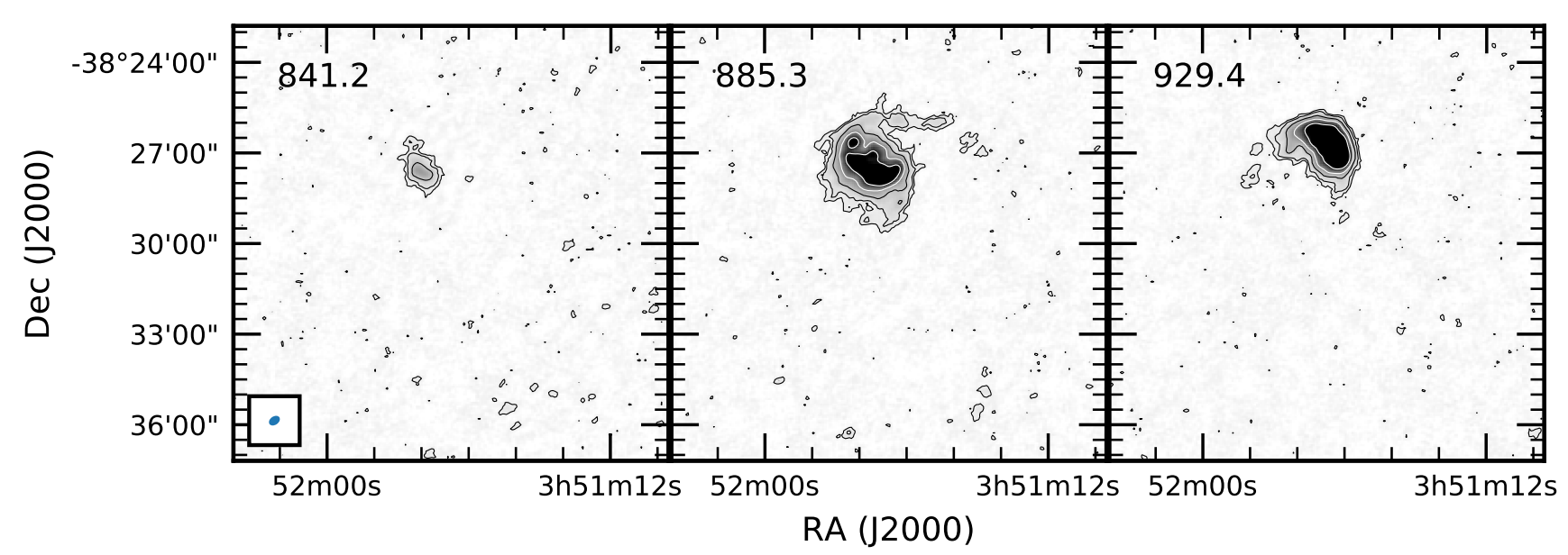

Fig. 3. Channel maps of the $4 \mathrm{k}$ data containing $\mathrm{H} \mathrm{I}$ emission produced using $r=1.0$. The velocity of each channel is given in the top left in $\mathrm{km} \mathrm{s}^{-1}$. The beam (as given in Table 2) is indicated in the box at the lower left of the left-hand panel. The contour levels are drawn at $(2.5,5.0,12.5) \sigma$ for the black contours, and at $(25,50) \sigma$ for the white contours. The noise $\sigma$ equals $86 \mu \mathrm{Jy}_{\text {beam }}{ }^{-1}$.

feature can also be seen in Figs. 2 and 3 as well as in the $r=1.5$ integrated $\mathrm{H}$ i image given below. We discuss the possible nature of these features below. Finally, the faint clouds to the south can also be seen in the $32 \mathrm{k}$ data, most clearly in the channel map at $892.5 \mathrm{~km} \mathrm{~s}^{-1}$.

\subsection{Moment maps}

Figure 5 shows the integrated H I maps (zeroth-moment maps) derived from the $4 \mathrm{k}$ and the $32 \mathrm{k}$ data for the four robust values discussed here. Small robust values emphasise high-resolution details in the disc, while the larger robust values (which are closer to natural weighting) emphasise the more extended lowcolumn density H I distribution.

The moment maps were created using the SoFiA source finder (Serra et al. 2015). We again searched for emission brighter than $3 \sigma$ using various smoothing kernels. The $4 \mathrm{k}$ data cubes were searched twice: once at their original resolution, and once with a Gaussian spatial smoothing kernel with a full width at half maximum (FWHM) that is equal to the beam size. Due to the limited number of channels no additional velocity smoothing kernels were used here.

The $32 \mathrm{k}$ cubes were searched four times each using combinations of the original spatial and velocity resolution, along with a Gaussian spatial kernel equal to the beam size and a boxcar velocity smoothing kernel of three channels. Larger kernels did not result in qualitatively better maps. The built-in comparison between positive and negative detections with a reliability threshold of 0.90 was used for both data sets to distinguish between noise peaks and real emission. For all cases, we consider the column density limit of the moment maps to be $3 \sigma$ in one channel.

In Fig. 5, the 32k data reveal additional details not seen in the $4 \mathrm{k}$ data set despite the somewhat shorter integration time of the former (cf. Table 1). This increased column density sensitivity is due to the $32 \mathrm{k}$ channel width being better matched to the width of the H I profiles (typical FWHM values are $\lesssim 20 \mathrm{~km} \mathrm{~s}^{-1}$ ) than the $4 \mathrm{k}$ channels.

The $r=0.0$ maps show a regular disc, with only slight indications of the extension to the west. The disc becomes more and more asymmetric and lopsided towards higher $r$ values. The $r=1.0$ and $r=1.5$ maps clearly show the western filament, while towards the northern edge the column density contours are much closer together than towards the southern edge. The change from a regular disc to the more asymmetrical outer parts occurs around $\sim 1 \times 10^{20} \mathrm{~cm}^{-2}$.

The western filament shows a number of kinks or breaks in its morphology and extends further east than the moment maps suggest. The channel maps between 870.5 and $903.6 \mathrm{~km} \mathrm{~s}^{-1}$ in Fig. 4 show that it connects with the north-eastern part of the disc. In the moment maps this is, however, hidden by superimposed bright $\mathrm{HI}$ emission from lower velocities. As the channel maps at $870.5 \mathrm{~km} \mathrm{~s}^{-1}$ and $881.5 \mathrm{~km} \mathrm{~s}^{-1}$ are also the ones where the southern extension is most prominently visible, it is possible that the western filament and the southern extension of the disc are related.

Figure 6 shows an overlay of the $r=0.5$ integrated H I map on the SINGG $R$-band and $\mathrm{H} \alpha$ data. The filament and southern extension extend well beyond the optical disc, as expected. The $R$-band image shows no optical counterpart down to the limiting surface brightness of $27.6 \mathrm{AB}$ mag $\operatorname{arcsec}^{-2}$. In the $\mathrm{H} \alpha$ image we also do not see evidence for recent star formation at these locations. The two prominent young star clusters are located at an under-density in the HI. We will return to these clusters in the next section. DECaLS images show a faint object close to the tip of the filament at $(\alpha, \delta)=03^{\mathrm{h}} 51^{\mathrm{m}} 20^{\mathrm{s}},-38^{\circ} 25^{\prime} 35^{\prime \prime}$. It appears in both $g$ and $r$ bands, so ensuring that the object is real. We discuss this object further in Sect. 5.2.

The intensity-weighted mean velocity fields (shown in Fig. 7) paint a similar picture. The inner disc shows a very regular overall velocity field, with only small $\left(\sim 5-10 \mathrm{~km} \mathrm{~s}^{-1}\right)$ perturbations (visible in the high-resolution data) that are consistent with turbulence and star-formation related non-circular motions.

The velocities of the extension differ significantly $\left(\sim 50-60 \mathrm{~km} \mathrm{~s}^{-1}\right)$ from those in the northern part of the disc and are more consistent with those found in the southern part, potentially hinting at a connection between the western filament and the southern extension.

The kinematic center seems to be offset from the center of the bright $\mathrm{H}$ I emission. We note that the kinematic minor axis is not precisely perpendicular to the kinematic major axis, suggesting the presence of a non-axisymmetric structure in the main disc of the galaxy. However, the photometric position angle of the disc is difficult to determine. There is a difference of $\sim 20^{\circ}$ between the major axis position angles of the mid-infrared Spitzer data at 3.6 and $4.5 \mu \mathrm{m}$ (cf. archival data of Muñoz-Mateos et al. 2015), 


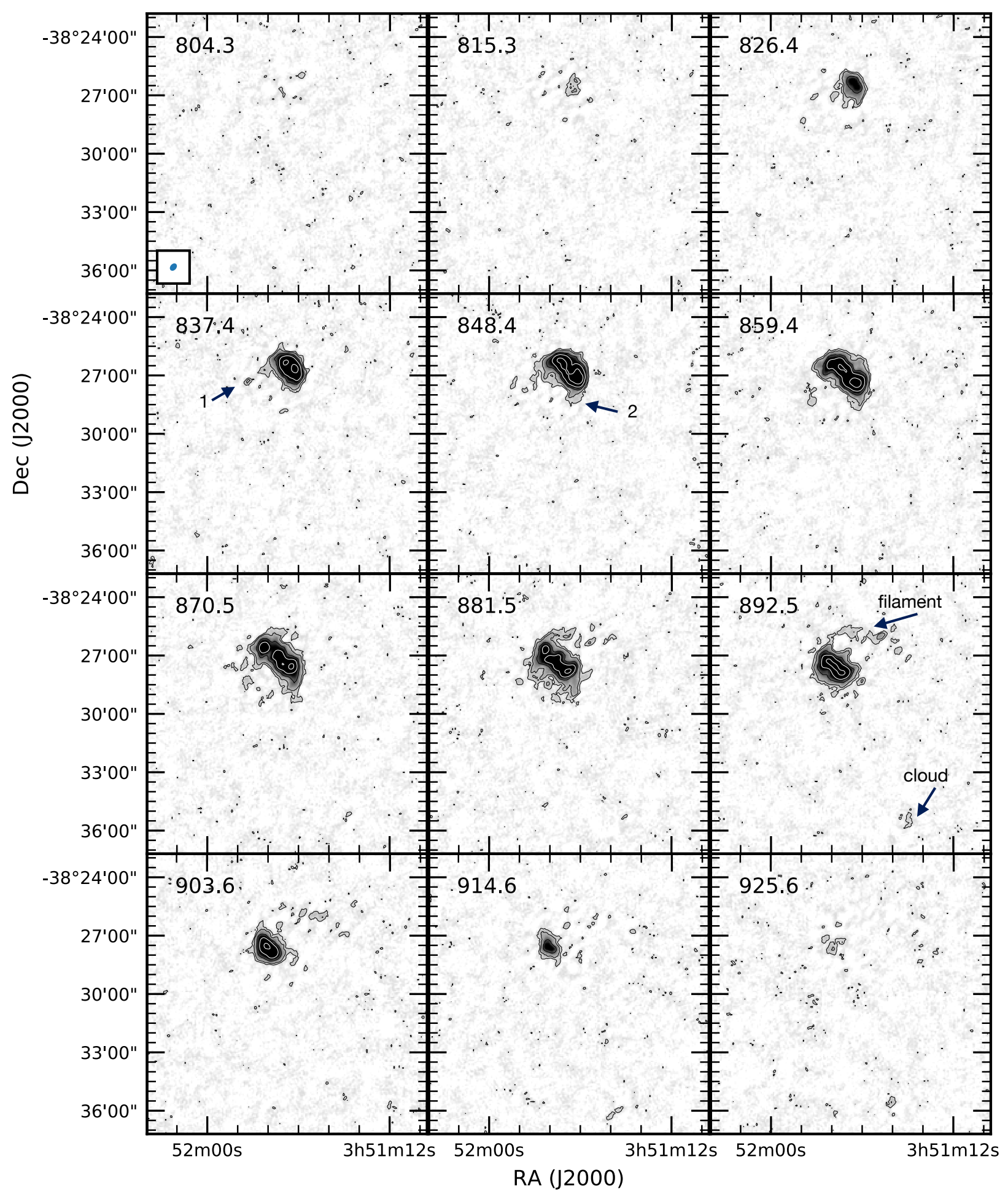

Fig. 4. Channel maps of the 32k data containing H I emission produced using $r=1.0$. Only every second channel is shown. The velocity of each channel is given in the top left in $\mathrm{km} \mathrm{s}^{-1}$. The beam (as given in Table 2) is indicated in the box in the top-left panel. The contour levels are drawn at $(2.5,5.0,12.5) \sigma$ for the black contours, and at $(25,50) \sigma$ for the white contours. The noise $\sigma$ equals $255 \mu \mathrm{Jy}_{\text {beam }}{ }^{-1}$. The cloud and filament are indicated. Numbers " 1 " and " 2 " refer to features described in the text.

with the latter being close to the kinematic position angle, but these images are rather faint. Deeper near infrared imaging is necessary to get a better understanding of the structure of the stellar disc of this galaxy.

The second-moment map shows a region of high velocity dispersion in the central part of the disc, extending towards the east. This corresponds with the star formation regions just to the north of the optical center and also includes the two young star clusters in the eastern part of the disc. The high values at the northern and extreme eastern edge of the disc correspond to locations where the filament crosses the main body of the H I disc. These values are, therefore, due to the double velocity profiles along the line of sight, rather than a physical velocity dispersion. 


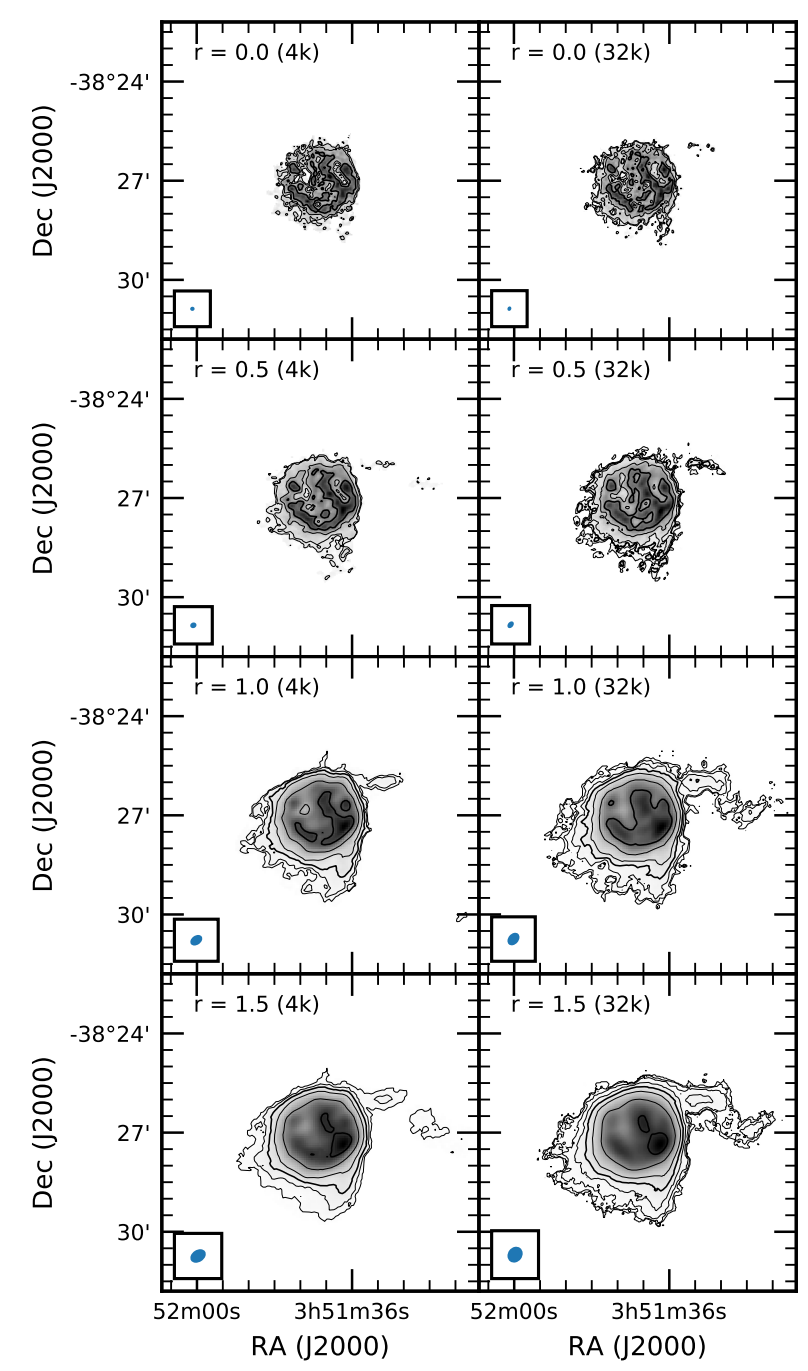

Fig. 5. Integrated MeerKAT H I (zeroth moment) maps of the galaxy ESO 302-G014 produced using different robust values. Rows show data imaged with different robust factors, given in the top-left of each panel, with values from top to bottom $r=0.0,0.5,1.0,1.5$. Columns show the two data sets, with the $4 \mathrm{k}$ data on the left and the $32 \mathrm{k}$ data on the right. The respective beams are indicated in the box in the lower-left corners of the panels, and are also listed in Table 2. The lowest contour levels shown are always the $3 \sigma$ contour levels, with $\sigma$ given in Table 2, followed by contours at fixed approximately logarithmically-spaced column density levels. This results in the following contour values (in units of $\left.10^{20} \mathrm{~cm}^{-2}\right)$ : left column (4k data), from top to bottom: $r=0.0:(2.9$, 5.0, 10.0); $r=0.5:(1.2,2.0,5.0,10.0) ; r=1.0:(0.3,0.5,1.0,2.0,5.0$ $10.0) ; r=1.5:(0.2,0.5,1.0,2.0,5.0,10.0)$. Right column: (32k data), from top to bottom: $r=0.0:(1.2,2.0,5.0,10.0) ; r=0.5:(0.4,1.0,2.0$, 5.0, 10.0); $r=1.0:(0.1,0.2,0.5,1.0,2.0,5.0,10.0) ; r=1.5:(0.06,0.1$, $0.2,0.5,1.0,2.0,5.0,10.0)$. The $10^{20} \mathrm{~cm}^{-2}$ and $10^{21} \mathrm{~cm}^{-2}$ contours are drawn with slightly thicker lines.

\subsection{Fluxes and total HI masses}

Table 2 also lists the total fluxes derived from the moment maps and the corresponding $\mathrm{HI}$ masses. The integrated flux spectrum (global profile) is shown for the 32k data in Fig. 8 for two robust values. The highest robust value recovers slightly more flux over the entire velocity range.

Uncertainties in the integrated fluxes and H I masses were derived for the MeerKAT- 16 and 32k data using the prescription given in Koribalski et al. (2004). We do not give uncertainties

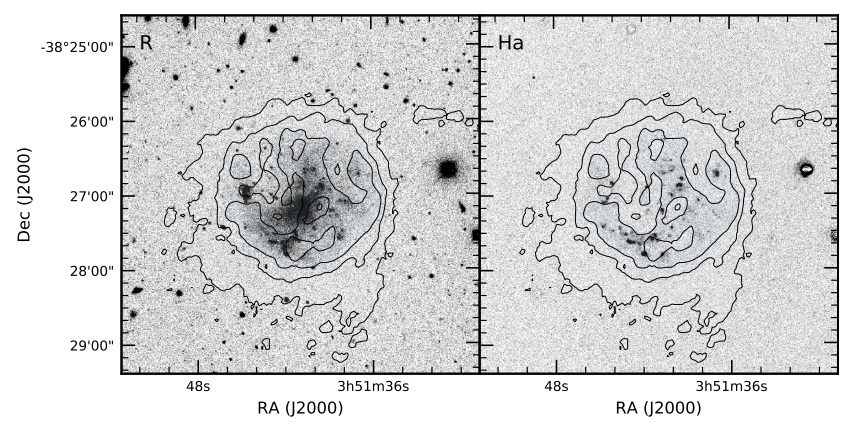

Fig. 6. Overlays of the MeerKAT $r=0.5$ integrated H I map on SINGG $R$-band (left panel) and $\mathrm{H} \alpha$ (right panel) images. For clarity, only a limited number of column density contours are shown. These are $(1.0,5.0,10.0) \times 10^{20} \mathrm{~cm}^{-2}$. Darker shading indicates the highest column densities.

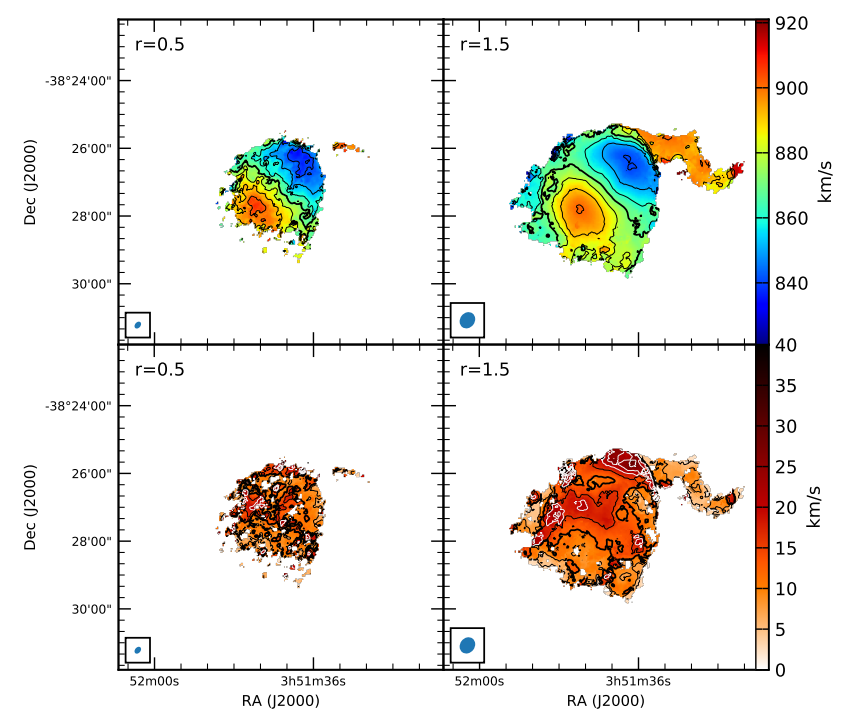

Fig. 7. First- and second-moment maps of the galaxy ESO 302-G014 based on the MeerKAT 32k data. Top row: intensity-weighted mean velocity fields, bottom row: velocity dispersion maps. Left column: $r=0.5$ map, right column: $r=1.5$ version. An intensity cut has been applied to the maps. Left column: only velocities where the column density is above $1 \times 10^{20} \mathrm{~cm}^{-2}$. Right column: the limit is $1 \times 10^{19} \mathrm{~cm}^{-2}$. Top row: the thick contour denotes a velocity of $871 \mathrm{~km} \mathrm{~s}^{-1}$ (the systemic velocity). From there contours are shown in intervals of $10 \mathrm{~km} \mathrm{~s}^{-1}$. Bottom row: the thick contour indicates $12 \mathrm{~km} \mathrm{~s}^{-1}$. Contour values change in intervals of $4 \mathrm{~km} \mathrm{~s}^{-1}$. The highest values are indicated by white contours.

for the $4 \mathrm{k}$ data due to the large channel width and resulting small number of channels containing emission.

We can compare these values with the integrated fluxes (and H I mass) derived from single-dish observations. The flux value listed in the HIPASS Bright Galaxy Catalogue is $11.2 \pm$ $2.0 \mathrm{Jy} \mathrm{km} \mathrm{s}^{-1}\left(3.6 \times 10^{8} M_{\odot}\right)$ (Koribalski et al. 2004). This value is derived using the HIPASS HI position. For this work, we have gone back to the HIPASS data and re-extracted the integrated spectrum using the optical position. We find a value of $11.4 \pm 0.2 \mathrm{Jy} \mathrm{km} \mathrm{s}^{-1}\left(3.7 \times 10^{8} M_{\odot}\right)$. This updated spectrum is shown in Fig. 8. We note the good agreement with the MeerKAT data.

ESO 302-G014 was also observed using the Green Bank Telescope (GBT). This observation was first presented in Sorgho et al. (2019), where a flux of $10.4 \pm 0.4 \mathrm{Jy} \mathrm{km} \mathrm{s}^{-1}\left(3.4 \times 10^{8} M_{\odot}\right)$ was derived. This was derived from masked data, so it should be 


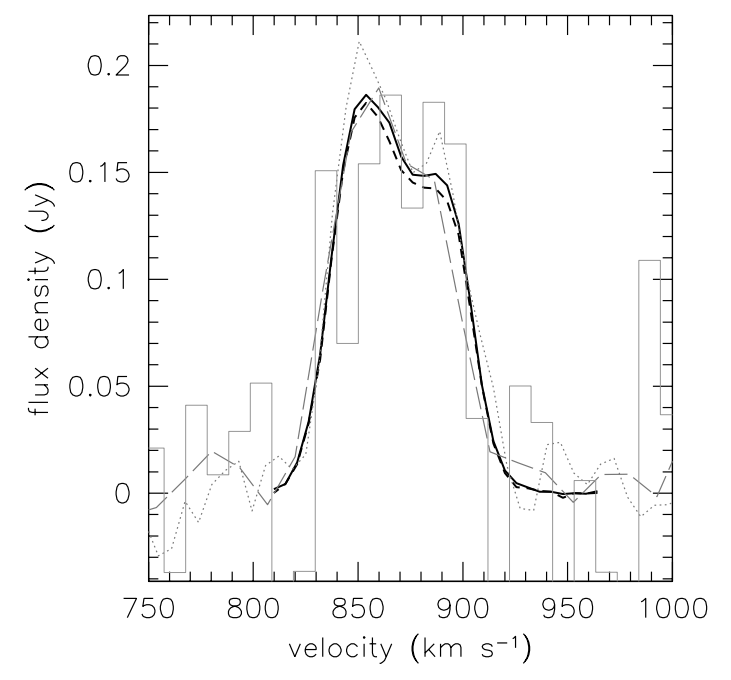

Fig. 8. Integrated $\mathrm{HI}$ and $\mathrm{CO}$ spectra (global profiles). The full curve shows the $r=1.5 \mathrm{HI}$ profile, the dashed curve the $r=0.5$ profile from MeerKAT. The dotted gray line is the H I global profile derived from GBT data (Sardone et al., in prep.). The long-dashed gray line is the HIPASS spectrum. The histogram indicates the global ALMA CO spectrum where the $\mathrm{CO}$ flux density values have been scaled down by a factor of three.

considered a lower limit. These data were re-analysed in Sardone et al. (in prep.), where a flux of $13.7 \mathrm{Jy} \mathrm{km} \mathrm{s}^{-1}\left(4.4 \times 10^{8} M_{\odot}\right)$ was found. This integrated spectrum is also shown in Fig. 8. In general, the agreement is good, except for the two peaks of the profile, where the GBT finds more flux. The angular size of the galaxy compared to the length and large number of short baselines of the MeerKAT data gives no reason for us to suspect that these are affected by zero-spacing issues. Rather, as in terms of column-density sensitivity the GBT observations are close to an order of magnitude deeper, it is possible that these peaks indicate the presence of a few times $10^{7} M_{\odot}$ of additional $\mathrm{HI}$ between $\sim 10^{17}$ and $\sim 10^{18} \mathrm{~cm}^{-2}$. The full MHONGOOSE data set of this galaxy should be able to confirm the presence of this low-column density H I.

\subsection{Clouds}

The $4 \mathrm{k}$ observations showed the presence of faint $\mathrm{H}$ I clouds $\sim 7^{\prime}$ south of the main body of the galaxy in the central channel map. A smoothed version of that channel map (left panel of Fig. 9) shows this more clearly. This is based on the $r=1.54 \mathrm{k}$ data smoothed to $45^{\prime \prime}$.

The complex is also visible in individual channel maps of the $32 \mathrm{k}$ data; compare with the channel map at $892.5 \mathrm{~km} \mathrm{~s}^{-1}$ in Fig. 4. Smoothing the $32 \mathrm{k}$ data also enhances the visibility of this complex. The right-hand panel in Fig. 9 shows a zeroth-moment map based on $45^{\prime \prime}, r=1.532 \mathrm{k}$ data, constructed using the same set of thresholds and kernels as used for the moment maps shown in Fig. 5.

The cloud is somewhat resolved ( $2-3$ beams) but has a low column-density overall. Its peak value reaches only $1.8 \times$ $10^{19} \mathrm{~cm}^{-2}$ in the $45^{\prime \prime}$ map. In the full-resolution, $r=1.5$, moment map, the cloud is not included due to the stringent criteria used. If these are relaxed, the cloud is detected with a peak column density of $4 \times 10^{19} \mathrm{~cm}^{-2}$. Nevertheless, the reality of the cloud is not in doubt due to its detection in the channel maps of two independent data sets.
The mass of the cloud as detected in the $45^{\prime \prime}$ data is $9.3 \times$ $10^{5} M_{\odot}$. This should be compared with the $45^{\prime \prime}$ resolution total H I mass of the galaxy of $3.8 \times 10^{8} M_{\odot}$, so the cloud contains only about $0.25 \%$ of the total $\mathrm{H}$ I mass. In comparison, the $\mathrm{H}$ I mass of the western filament is $\sim 4.5 \times 10^{6} M_{\odot}$, and given its morphology it is possible this filament consists of a number of similar lowmass clouds.

Figure 9 also shows an overlay of the smoothed moment map on a MeerLICHT $q$-band image. MeerLICHT is a wide-field $65 \mathrm{~cm}$ optical telescope located at the South African Astronomical Observatory in South Africa that observes any of MeerKAT's targets in a number of optical bands simultaneously with the MeerKAT observations. This overlay shows no optical counterparts for the filament and cloud at least down to the surface brightness levels of the ESO 302-G014 main disc. The deeper DECaLS images show no evidence for an optical counterpart of the cloud.

\section{Discussion}

The HI moment maps in Fig. 5 show a changing morphology for ESO 302-G014 when going from high to low column densities. At high column densities the disc is regular and appears almost unperturbed, with just a slight hint of the presence of the western filament. This changes dramatically when column densities that are an order of magnitude lower are probed. The western filament has become more prominent and in comparing the northern and southern edges of the disc, we can see a northern disc edge that is more compressed than the southern edge.

The central positions of the stellar and H I components do not coincide either. The centroid of the bright $\mathrm{H}$ I emission (the axi-symmetric inner part of the H I disc) lies $\sim 12^{\prime \prime}$ or $\sim 680 \mathrm{pc}$ to the north of the center of the stellar component (cf. left panel of Fig. 6).

Lopsidedness or asymmetries are common in disc and dwarf galaxies. They can manifest themselves as morphological lopsidedness, but also as kinematical lopsidedness, or as asymmetries in the integrated H I spectra. For an extensive overview, see the review by Jog \& Combes (2009) and references therein. Jog \& Combes (2009) note that at least $30 \%$ of disc galaxies show a lopsided stellar component, but that this frequency is higher in H I. For example, Richter \& Sancisi (1994) found that half of the 1700 galaxies they studied showed a strong lopsidedness in their global H I profiles. A recent study by Watts et al. (2020) of 562 galaxies finds a proportion of 37\%. Espada et al. (2011) studied lopsidedness in $\mathrm{H}$ I and distinguished between isolated and field galaxies. They find that only $\sim 2 \%$ of isolated galaxies show significant asymmetries, while this rate is much higher $(\sim 10-20 \%)$ in regular field galaxies.

There have been many attempts to explain lopsidedness using a variety of scenarios. Of the ones that are relevant here, the most promising ones are minor tidal interactions, the accretion of gas from the intergalactic medium, an off-centre disc within its halo, and ram pressure stripping (Jog \& Combes 2009). Reynolds et al. (2020) find a trend of increasing asymmetry strength with density of the environment, as also indicated by Espada et al. (2011). It is thus likely that ram pressure stripping and tidal interactions are the most likely scenarios in the higher density environments, with gas accretion explaining asymmetries seen in more isolated galaxies. We discuss a number of these scenarios and their relevance for ESO 302-G014 in more detail below. 


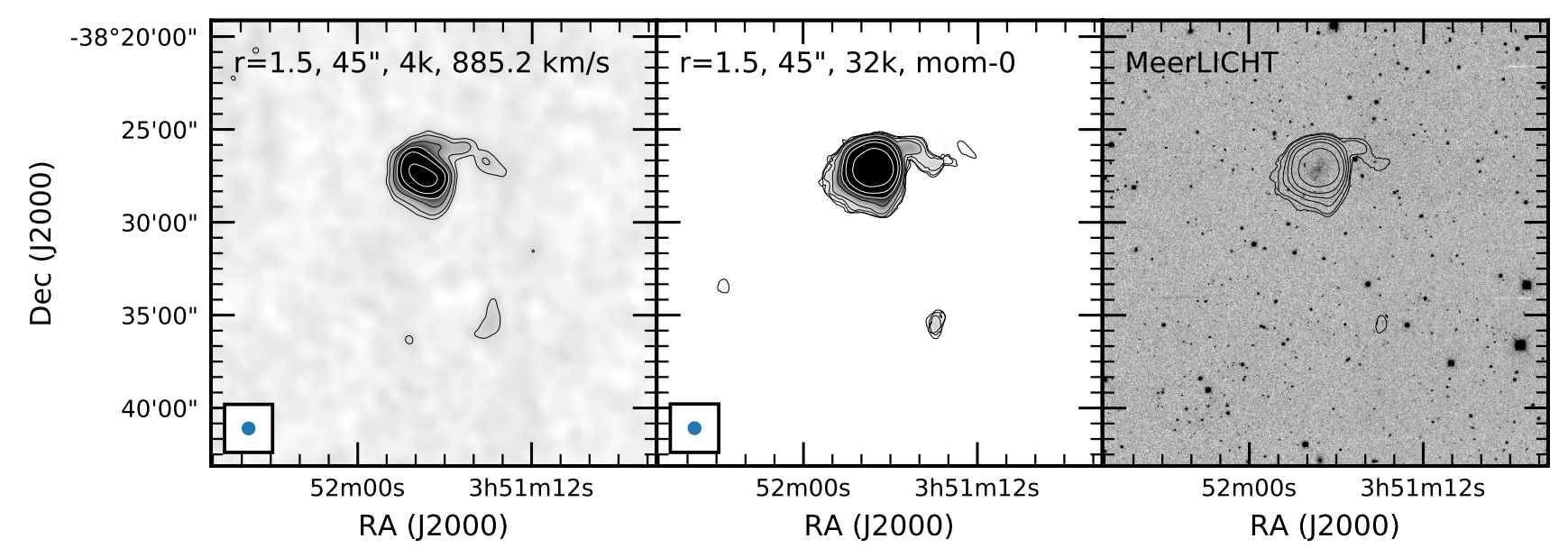

Fig. 9. Lower resolution $45^{\prime \prime}$ maps based on the $r=1.5$ data. Left panel: $4 \mathrm{k}$ data channel map at $885.2 \mathrm{~km} \mathrm{~s}^{-1}$. Contour levels are $(0.1,0.2,0.5$, $1.0,2.0,5.0) \times 10^{20} \mathrm{~cm}^{-2}$. Middle panel: $32 \mathrm{k}$ data integrated $\mathrm{H} \mathrm{I}$ (zeroth moment) map. Contour levels are $(0.03(3 \sigma), 0.05,0.1,0.2,0.5,1.0,2.0$, $5.0) \times 10^{20} \mathrm{~cm}^{-2}$. Right panel: MeerLICHT $q$-band optical image, with $32 \mathrm{k}$ integrated H I map as overlay. Contour levels are $(0.1,0.2,0.5,1.0,2.0$, $5.0) \times 10^{20} \mathrm{~cm}^{-2}$.

\subsection{Ram pressure stripping scenario}

Looking at the large-scale environment of ESO 302-G014, we find that (in projection) it is located in the vicinity of the Fornax cluster, at the northern end of a large-scale structure filament that connects the Dorado group with the Fornax cluster (see Fig. 1 in Venhola et al. 2018). The projected angular distance to the centre of the main component of the Fornax cluster (NGC 1399) is $\sim 4.0^{\circ}$.

ESO 302-G014 is, however, significantly closer to us than Fornax and at the distance of ESO 302-G014 this projected distance corresponds to $0.8 \mathrm{Mpc}$. Using the radial velocities of NGC $1399\left(1425 \mathrm{~km} \mathrm{~s}^{-1}\right)$ and ESO 302-G014 $\left(871 \mathrm{~km} \mathrm{~s}^{-1}\right)$ as distance indicators, we find a line-of-sight distance of $8.6 \mathrm{Mpc}$. For comparison, the virial radius of the Fornax cluster is $0.7 \mathrm{Mpc}$ (Drinkwater et al. 2001). A recent catalogue of nearby groups (Kourkchi \& Tully 2017) confirms that ESO 302-G014 is not a member of Fornax nor of any of the groups in the Fornax region. It is, therefore, unlikely that ram pressure stripping due to infall in Fornax can explain the morphology of the galaxy.

It is, however, suggestive that the compression of the northern edge of the disc points towards the Fornax cluster. Is it therefore possible that ram pressure due to the IGM between Fornax and the Dorado group can cause the observed morphology?

Motivated by observations of NGC 300 in the Sculptor group, Westmeier et al. (2011) show that for a galaxy moving at $200 \mathrm{~km} \mathrm{~s}^{-1}$, the density of the IGM needs to be $\sim 10^{-5} \mathrm{~cm}^{-3}$ for ram stripping to occur, comparable with the density of the Local Group medium. This density is similar to the estimate by Bureau \& Carignan (2002), who studied the effect of ram pressure on the H I distribution of Holmberg II, a dwarf galaxy falling into the M 81/M 82/NGC 3077 group.

These two case studies show that the minimum IGM densities needed for ram pressure stripping to occur are those typically found in a group environment. Simulations by Pilkington et al. (2011) of a Holmberg II-like dwarf galaxy suggests that a lopsidedness with an apparent "compression" on one side can also be caused by internal processes. The presence of the western extension is also not readily explained by ram pressure stripping. In summary, ram pressure stripping is, therefore, not expected to play a major role in defining the H I morphology of ESO 302G014.

\subsection{Interaction and accretion scenario}

Since ram pressure stripping is unlikely to explain the H I morphology of the galaxy, we consider the possibility that its origin lies in the infall of a smaller galaxy. The H I mass of the western filament as visible in the moment maps is $\sim 4.5 \times 10^{6} M_{\odot}$. Taking into account that these integrated maps do not show the eastern part of the extension, the total H I mass of the extension is about a factor of two larger. Assuming that all this H I originated in the infalling galaxy, then this would have been a $\sim 10^{7} M_{\odot}$ dwarf galaxy.

As described in Sect. 4, the features in the northern part of the disc unwind in the same sense as the southwestern one (feature 2 in Fig. 4). These two together form a two-armed spiral-like feature that would be driven by a small companion or satellite. This could have either already merged, or be linked to one or more of the gas clouds, or to some clump in the galaxy itself, that is, on its way to a merging. In this case, the closeness of the iso-density contours in the northern part of the galaxy would be linked to the higher density of the northern arm, compared to the southern one.

A second interesting feature in the data suggesting an interaction or accretion scenario is the cloud to the south of ESO $302-$ G014. It has a central velocity of $892 \mathrm{~km} \mathrm{~s}^{-1}$ with a velocity width $W_{50} \simeq 25 \mathrm{~km} \mathrm{~s}^{-1}$. This recessional velocity agrees well with that of the western filament (cf. Fig. 4). The left panel in Fig. 9 shows the filament curving south, and extending this curvature, an arc can be drawn that connects the filament with the cloud. This suggests that all these features may be related. A careful study of the MeerKAT data of this area did not show any signs of the connecting $\mathrm{HI}$, but this may simply mean that it is either of even lower column-density, or partly ionised. The fulldepth MHONGOOSE data should be able to constrain this much better.

The data are not deep enough or resolved enough to study the dynamics of the cloud, but it is unlikely to be a dwarf galaxy. Its H I mass is only slightly less than that of Leo T, the lowest mass gas-rich galaxy currently known with an Hi mass of $4.1 \times 10^{5} M_{\odot}$ (Adams \& Oosterloo 2018). The peak column densities in the cloud are, however, a factor of ten lower than those found in Leo T (even though spatial resolutions are similar). The cloud measures $\sim 3.5 \mathrm{kpc}$ along its minor axis, while Leo $\mathrm{T}$ has 

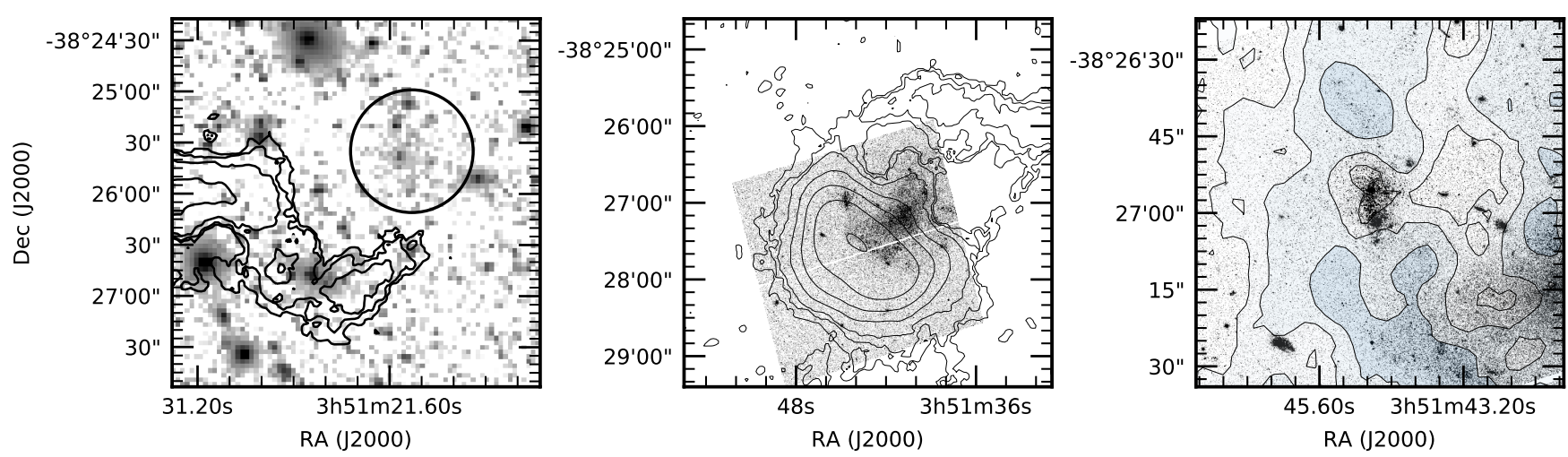

Fig. 10. Left panel: overlay of the $r=1.5,32 \mathrm{k}$ integrated H I map on a combined $g+r$ image from DECaLS, binned to a pixel size of $2.7^{\prime \prime}$ to increase signal-to-noise. Contour levels are as in Fig. 5. The circle indicates the dwarf galaxy candidate. Middle panel: contours show the zeroth-moment map of emission $>2 \sigma$ in the three channels between 892.5 and $903.6 \mathrm{~km} \mathrm{~s}^{-1}$ in the $r=0.532 \mathrm{k}$ cube. Levels are $(2.5,5,10,25,50,100,250) \cdot \sigma_{\text {mom }}$, where $\sigma_{\text {mom }}=1.3 \times 10^{19} \mathrm{~cm}^{-2}$. Right panel: zoom-in of the HST image centered on the stellar clusters. Contours represent the $r=0.0$ zerothmoment map. Contour levels are $(2.0,5.0,10.0) \times 10^{20} \mathrm{~cm}^{-2}$.

an $\mathrm{H}$ I diameter of $\sim 0.8 \mathrm{kpc}$ measured at similar column density levels. The cloud is therefore an altogether more diffuse entity than Leo T. Furthermore, neither the MeerLICHT image shown in Fig. 9, nor the DECaLS images show evidence for an optical counterpart.

As noted above, the centers of the stellar distribution and that of the inner (high column density) H I disc do not coincide. The position of the center of the brighter parts of the H I image is offset with respect to the center of the stellar component (by $\sim 12^{\prime \prime}=680 \mathrm{pc}$ to the north). Such a mismatch has been discussed in (amongst others) a paper by Pardy et al. (2016). They used numerical simulations to study the interaction of an LMC-sized barred irregular galaxy with a small companion with a mass ratio of 1:10, and found that the dynamical center should coincide with the center of the bar, rather than the center of the H I distribution. While ESO 302-G014 shows no pronounced bar, the tidal effects we now witness may have been due to a similar, earlier interaction with a much smaller, dwarf companion, which by now has been captured, damping the amplitude of the bar in the process (e.g. Athanassoula 1996).

The deep DECaLS images mentioned in Sect. 2 show a faint and diffuse object close to the tip of the filament with properties consistent with those of a dwarf galaxy (Fig. 10, left panel). A Sersic model fit gives an effective radius of $0.71 \mathrm{kpc}$, a Sersic index of 0.77 , a $g-r$ color of $0.37 \mathrm{mag}$ (no extinction correction applied), and an absolute magnitude of $M_{g}=-10.0$, all assuming that the object is at the same distance as ESO 302G014. These properties are consistent with those of a diffuse dwarf galaxy. It is worth noting that the object is extremely faint, with a mean surface brightness within the effective radius in the $g$-band of $\left\langle\mu_{g}\right\rangle=27.8 \mathrm{mag} \operatorname{arcsec}^{-2}$. Its slightly blue colours make it less likely that it is a background galaxy. While no $\mathrm{HI}$ emission is seen at this position in the integrated H I maps, inspection of the $r=1.532 \mathrm{k}$ cube shows a $3 \sigma$ peak close to the optical position but only in a single channel. The forthcoming deeper MHONGOOSE observations should show whether this H I signal is real and whether we can associate this potential dwarf with ESO 302-G014. However, even if confirmed, it is not clear that this optical dwarf would be responsible for both the tail and the southern cloud. Thus, a more complex explanation would still be needed.

Currently, the nearest major galaxy to ESO 302-G014 is ESO 302-G009 (HIPASS J0347-38), at a projected distance of $\sim 180 \mathrm{kpc}$. It is more massive and slightly more distant (Meurer et al. 2006 give a distance of 13.4 Mpc for ESO 302-G009). The deep GBT observations presented in Sorgho et al. (2019) and Sardone et al. (in prep.) show no evidence of H I features between the two galaxies and it is unlikely that tidal interactions between these two galaxies can cause the more small-scale features that we observe around ESO 302-G014.

Bournaud et al. (2005) also studied lopsidedness in galaxies. They found that (in the near-infrared) out of a sample of 149 disc galaxies, two-thirds have significant lopsidedness. Late-type galaxies tend to be more lopsided in their study. Bournaud et al. (2005) presented simulations that they use to constrain the origins for lopsidedness. One of the conclusions is that any tidal interaction or merger can only occur with a small companion of a mass ratio of 10:1 or more. More massive companions would transform the main galaxy towards an elliptical galaxy.

A second scenario, one explored by Bournaud et al. (2005), is asymmetrical accretion from a cosmic gas filament. They found that this kind of accretion can result in strongly lopsided discs, even showing arm-like features. A direct comparison with our data is not straightforward, as the Bournaud et al. (2005) simulations model the stellar population, not the $\mathrm{HI}$ disc. However, as the $\mathrm{HI}$ is generally a more sensitive tracer of interactions than the stellar population (e.g. Chakrabarti et al. 2011), it is likely that the effects described in Bournaud et al. (2005) should also be visible in the H I, and possibly even more pronounced.

\subsection{A potential link with star formation}

As noted above, the integrated H I maps do not show the full extent of the western filament, as its eastern part is hidden by the bright emission of main-disc $\mathrm{HI}$ at different velocities. We created an integrated H I map of the emission between 892.5 and $903.6 \mathrm{~km} \mathrm{~s}^{-1}$, including all emission $>2 \sigma$ in each of the three channel maps. This map is shown in Fig. 10, where it is overlaid on one of the HST images discussed in Sect. 2.

The moment map shows the filament connecting with the main disc HI just to the north of two stellar clusters, which are the two brightest stellar condensations in the galaxy. These clusters are shown in more detail in the right panel of Fig. 10, where we have overlaid the highest resolution $r=0.0$ integrated H I map. An alternative presentation is shown in Fig. 11, which combines the MeerKAT image with the available HST imaging. 


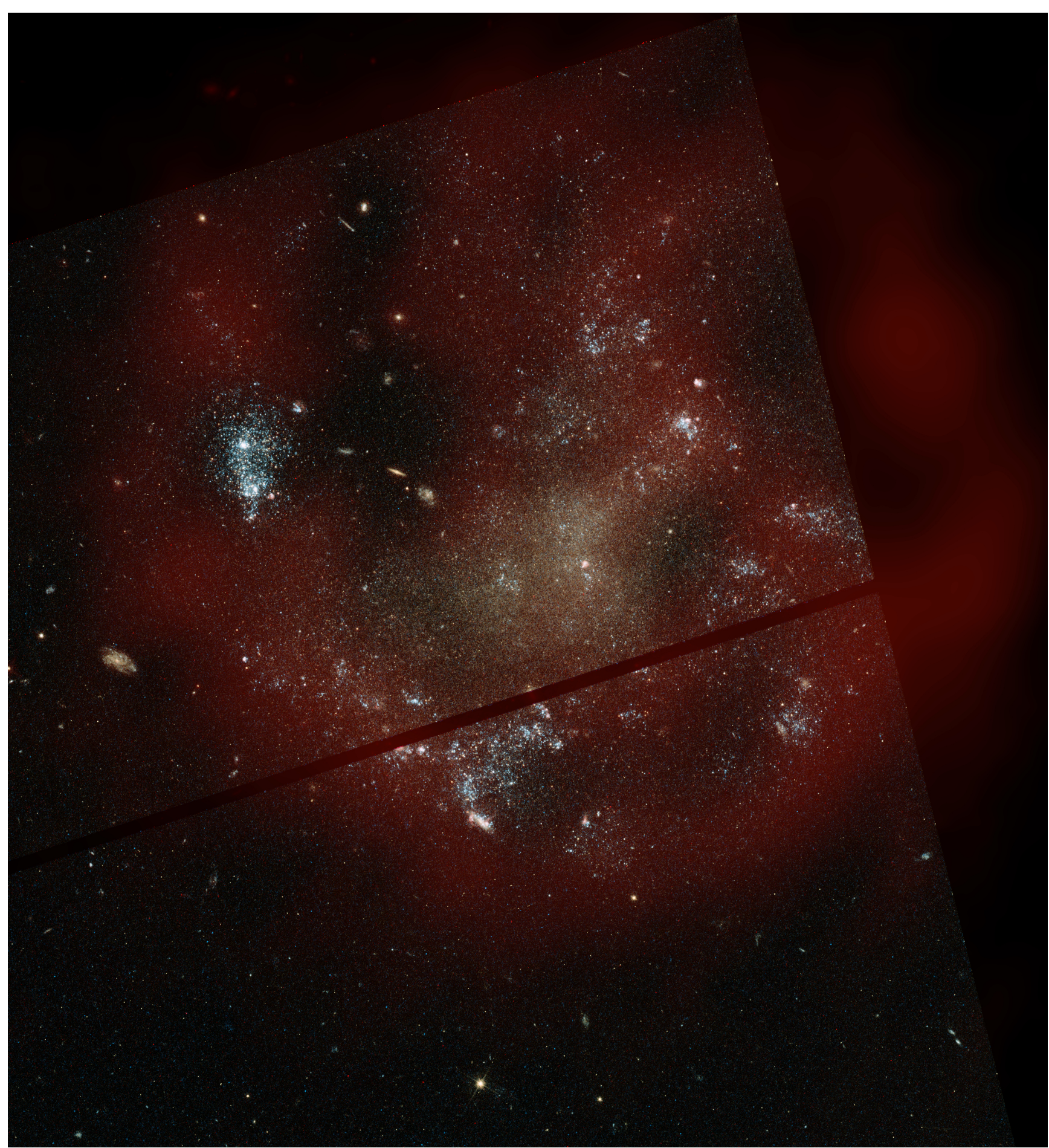

Fig. 11. Combination of the MeerKAT $r=0.5$ integrated H I map (red) overlaid on a colour combination of HST WFC3 IR $F 160 W$ (transparent red), HST ACS $F 658 N \mathrm{H} \alpha$ (red), HST ACS $F 606 W$ (green-yellow), and HST ACS $F 336 W$ (green-blue).

Both figures show a pronounced under-density in $\mathrm{HI}$ at the position of the clusters. This is the only place in the galaxy where we can find such prominent stellar clusters. One possible explanation is that this star formation is triggered by the filament connecting it to the main disc. Prieto et al. (2012) studied these clusters in detail and derived an age for these clusters of $\sim 9 \mathrm{Myr}$, with the corresponding ultra-violet-based SFR accounting for $\sim 30 \%$ of the total SFR of the galaxy.

The possibility of enhanced star formation is also suggested by the detection of molecular gas. Using the ALMA data described in Sect. 2, we find an integrated $\operatorname{CO}(J=1-0)$ flux $S$ (CO) $\mathrm{d} V$ of $32 \pm 5 \mathrm{Jy} \mathrm{km} \mathrm{s}^{-1}$. The integrated CO profile is shown in Fig. 8. Using Eq. (3) from Solomon \& Vanden Bout (2005) and a standard $\mathrm{CO}-$ to- $\mathrm{H}_{2}$ conversion factor, we find the molecular gas mass to be $4.6 \times 10^{7} M_{\odot}$, which is significant for a lowmass late-type $\mathrm{IB}(\mathrm{s}) \mathrm{m}$ galaxy with a luminosity of $\sim 10^{8} L_{\odot}$. The molecular gas mass almost equals the stellar mass, which is not commonly found in dwarf galaxies. However, the metallicity is low in these kind of low-mass galaxies, and the conversion factor is expected to be larger (e.g. Bolatto et al. 2013), resulting 

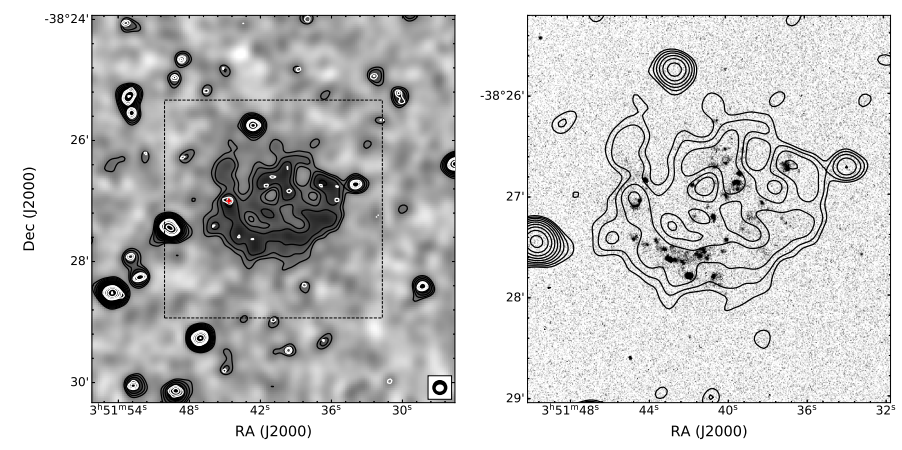

Fig. 12. Comparison between radio continuum and $\mathrm{H} \alpha$ emission. Left panel: grayscale shows the radio continuum image smoothed to $15^{\prime \prime}$ resolution, and the black contours are from the same map starting at $3 \sigma$ and increasing by powers of $\sqrt{2}$. White contours are for the full-resolution continuum image starting at $4 \sigma$ and increasing by powers of $\sqrt{2}$ up to $8 \sigma$ and then by powers of two thereafter. The beam sizes of the radio continuum images are shown in the bottom right corner, with colors corresponding to their contours. The position of SN2008jb is shown with a red cross. Right panel: $\mathrm{H} \alpha$ image from Meurer et al. (2006) in grayscale, within the region indicated by the dashed square in the left panel, and overlaid with the same $15^{\prime \prime}$-resolution radio continuum contours as in the left panel.

in a larger molecular mass. The molecular to atomic mass ratio is $\sim 0.12$ and is small compared to that found in spiral galaxies, but it is on the high end of the range found in dwarf irregular galaxies (Leroy et al. 2009; Young \& Scoville 1991). The similarity of the $\mathrm{CO}$ and $\mathrm{HI}$ global profiles suggests the presence of molecular gas throughout most of the disc.

It is therefore conceivable that the western extension is related to a minor interaction or merger, leading to the currently observed star formation and the significant molecular component along with contributing to the lopsidedness or asymmetry of the galaxy. More examples of the triggering of star formation in dwarf galaxies by minor interactions are discussed in Lelli et al. (2014). Sengupta et al. (2012) also discuss a case similar to that of ESO 302-G014. Their target galaxy, UGC 1547 (CIG 85), is an irregular galaxy, with one edge more compressed than the other, both in the H I and optical. It has a regular velocity field in the inner parts, is gas-rich and shows a recent increase in star formation. In this case, Sengupta et al. (2012) identify the potential remnants of the two dwarf galaxies responsible, showing that accretion of one or multiple satellites can indeed explain the enhanced star formation and asymmetries.

Further insights into the star formation history can be gleaned from the radio continuum image shown in Fig. 12. The morphology is most clearly traced at $15^{\prime \prime}(\sim 1 \mathrm{kpc})$ resolution. Here, we can see a ridge of prominent radio continuum around the periphery of the disc. The radio continuum emission is a variable combination of thermal and non-thermal (synchrotron) radiation, useful together as an indirect tracer of star formation; using the SFR calibration from Murphy et al. (2011), we estimate $\mathrm{SFR}_{1.4 \mathrm{GHz}}=0.09 \mathrm{M}_{\odot} \mathrm{yr}^{-1}$ (corrected to a Salpeter IMF). This is higher than the $\mathrm{H} \alpha$ and UV SFR discussed earlier, but this difference is most likely explained by the different star-formation time scales involved and by ignoring of dust-obscured star formation. An in-depth discussion of this SFR value is beyond the scope of this paper.

In most locations, we see an excellent correspondence between the bright continuum emission and $\mathrm{H} \alpha$ features, suggesting that the thermal fraction in those regions is relatively high. However, in the southwestern region, the radio continuum ridge diverges from the arc of $\mathrm{H} \alpha$-emitting regions. In that area, the radio continuum is more synchrotron-dominated. The characteristic timescale for synchrotron radiation at this frequency, for a typical magnetic field strength of $5 \mu \mathrm{G}$, is $10^{8} \mathrm{yr}$ (Condon 1992). These morphological features may indicate that star formation has progressed around the periphery of the disc, clockwise from the southwest region and proceeding most recently to the star-forming regions near SN2008jb. We consider whether such a sequential progression of star formation around the edge of the disc could have been spurred by the infall of an H I filament like the one currently seen to the west of ESO 302-G014. We anticipate that this picture can be tested further by mapping the continuum emission at another frequency and examining the variation in synchrotron spectral index, which is indicative of the characteristic age of the cosmic rays, in addition to carefully modelling the $\mathrm{HI}$ kinematics.

Finally, it is possible that some of the low-column density gas that we find is due to direct cold accretion from the IGM. Putman et al. (2012) suggest that the accreting gas is mostly ionised and only condenses into neutral gas close to the disc. At first glance, this seems consistent with the western filament. However, the densest parts of the filament have column densities of $\sim 5 \times 10^{19} \mathrm{~cm}^{-2}$, meaning these kind of clouds would have been detected at the sensitivity of HALOGAS. If accretion from the IGM into such high column density features is common, HALOGAS would have detected many more clouds. The fact that it has not (Kamphuis et al., in prep.) suggests that any pure accretion must take place at lower column densities and that the western filament is indeed better explained by a minor interaction or merger scenario.

\section{Summary}

In this paper, we present three MeerKAT H I commissioning observations of MHONGOOSE galaxy ESO 302-G014. At the highest column densities, the disc is regular and symmetric. Going to lower column densities, the disc becomes more lopsided and asymmetric. The northern edge of the disc looks compressed, while the southern edge is more diffuse.

The galaxy also exhibits an extension or tail of low-mass $\mathrm{HI}$ clouds, which could be connected with an isolated H I cloud $\sim 7^{\prime}$ $(\sim 23 \mathrm{kpc})$ to the south of the main galaxy. The tail and clouds could be the result of a minor interaction with a $\sim 10^{7} M_{\odot}$ dwarf galaxy, which could also explain the lopsidedness of the disc. Deep optical images show a faint potential dwarf galaxy near the tip of the tail, but a confirmation of its association with ESO $302-$ G014 will require deeper H I observations.

The galaxy has a significant molecular component. It is possible that this, and the presence of prominent stellar clusters, is related to a star formation event triggered by the interaction. The final, deeper MHONGOOSE observations should help explain some of these intriguing features.

These MHONGOOSE commissioning observations have shown that MeerKAT produces H I data of exquisite quality. Here, we highlight the capability to produce high-quality imaging over a large range in angular resolution. This is a great advantage that will help provide a more complete observational picture of the fate of neutral gas in galaxies as it moves from the IGM into galaxies.

Acknowledgements. It is a pleasure to thank the MeerKAT commissioning team without whom this paper would not have been possible. The MeerKAT telescope is operated by the South African Radio Astronomy Observatory, which is a facility of the National Research Foundation, an agency of the Department 
of Science and Innovation. (Part of) the data published here have been reduced using the CARACal pipeline, partially supported by ERC Starting grant number 679627 "FORNAX", MAECI Grant Number ZA18GR02, DST-NRF Grant Number 113121 as part of the ISARP Joint Research Scheme, and BMBF project 05A17PC2 for D-MeerKAT. Information about CARACal can be obtained online under the URL: https://caracal.readthedocs.io. This work is partly based on data obtained with the MeerLICHT telescope, located at the SAAO Sutherland station, South Africa. The MeerLICHT telescope is run by the MeerLICHT consortium, on behalf of Radboud University, the University of Cape Town, the Netherlands Foundation for Scientific Research (NWO), the National Research Facility of South Africa through the South African Astronomical Observatory, the University of Oxford, the University of Manchester and the University of Amsterdam. The work of PK is partially supported by the BMBF project 05A17PC2 for D-MeerKAT. This project has received funding from the European Research Council (ERC) under the European Union's Horizon 2020 research and innovation programme (grant agreement no. 679627; project name FORNAX). BKG acknowledges the UK's Science \& Technology Facilities Council (STFC), through the University of Hull Consolidated Grant (ST/R000840/1). KS acknowledges funding from the Natural Sciences and Engineering Council of Canada (NSERC). AS is supported by an NSF Astronomy and Astrophysics Postdoctoral Fellowship under award AST-1903834. EA and $\mathrm{AB}$ thank the CNES for financial support. The work of DJP was partially supported by NSF CAREER grant AST-1149491. FB acknowledges funding from the European Union's Horizon 2020 research and innovation programme (grant agreement no. 726384/EMPIRE). LVM and JR acknowledge financial support from the grants AYA2015-65973-C3-1-R and RTI2018-096228- B-C31 (MINECO/FEDER, UE), as well as from the State Agency for Research of the Spanish MCIU through the "Center of Excellence Severo Ochoa" award to the Instituto de Astrofísica de Andalucía (SEV-2017-0709).

\section{References}

Adams, E. A. K., \& Oosterloo, T. A. 2018, A\&A, 612, A26

Athanassoula, E. 1996, in IAU Colloq. 157: Barred Galaxies, eds. R. Buta, D. A Crocker, \& B. G. Elmegreen, ASP Conf. Ser., 91, 309

Bacchini, C., Fraternali, F., Iorio, G., \& Pezzulli, G. 2019, A\&A, 622, A64

Bigiel, F., Leroy, A., Walter, F., et al. 2008, AJ, 136, 2846

Bigiel, F., Leroy, A. K., Walter, F., et al. 2011, ApJ, 730, L13

Bolatto, A. D., Wolfire, M., \& Leroy, A. K. 2013, ARA\&A, 51, 207

Bournaud, F., Combes, F., Jog, C. J., \& Puerari, I. 2005, A\&A, 438, 507

Braun, R., Bourke, T., Green, J. A., Keane, E., \& Wagg, J. 2015, Advancing Astrophysics with the Square Kilometre Array (AASKA14), 174

Briggs, D. S. 1995, Am. Astron. Soc. Meet. Abstr., 187, 112.02

Bureau, M., \& Carignan, C. 2002, AJ, 123, 1316

Camilo, F. 2018, Nat. Astron., 2, 594

Chakrabarti, S., Bigiel, F., Chang, P., \& Blitz, L. 2011, ApJ, 743, 35

Cluver, M. E., Jarrett, T. H., Hopkins, A. M., et al. 2014, ApJ, 782, 90

Condon, J. J. 1992, ARA\&A, 30, 575

de Blok, W. J. G., Adams, E. A. K., Amram, P., et al. 2016, MeerKAT Science: On the Pathway to the SKA, 7

Dey, A., Schlegel, D. J., Lang, D., et al. 2019, AJ, 157, 168

Drinkwater, M. J., Gregg, M. D., \& Colless, M. 2001, ApJ, 548, L139

Elson, E. C., Kam, S. Z., Chemin, L., Carignan, C., \& Jarrett, T. H. 2019, MNRAS, 483, 931

Espada, D., Verdes-Montenegro, L., Huchtmeier, W. K., et al. 2011, A\&A, 532, A117

Fraternali, F. 2017, in Gas Accretion onto Galaxies, eds. A. Fox, \& R. Davé (Springer International Publishing AG), Astrophys. Space Sci. Lib., 430, 323

Fraternali, F., Marasco, A., Marinacci, F., \& Binney, J. 2013, ApJ, 764, L21

Heald, G., Józsa, G., Serra, P., et al. 2011, A\&A, 526, A118

Heiner, J. S., Allen, R. J., Emonts, B. H. C., \& van der Kruit, P. C. 2008, ApJ, 673,798

Holwerda, B. W., Pirzkal, N., \& Heiner, J. S. 2012, MNRAS, 427, 3159

Hu, W., Hoppmann, L., Staveley-Smith, L., et al. 2019, MNRAS, 489, 1619

Jáchym, P., Kenney, J. D. P., Sun, M., et al. 2019, ApJ, 883, 145

Jog, C. J., \& Combes, F. 2009, Phys. Rep., 471, 75

Jonas, J., \& MeerKAT Team 2016, MeerKAT Science: On the Pathway to the SKA, 1

Józsa, G. I. G., White, S. V., Thorat, K., et al. 2020, ArXiv e-prints [arXiv:2006.02955]

Kanekar, N., Sethi, S., \& Dwarakanath, K. S. 2016, ApJ, 818, L28

Kennicutt, R. C., Jr. 1998, ApJ, 498, 541

Kereš, D., Katz, N., Weinberg, D. H., \& Davé, R. 2005, MNRAS, 363, 2

Koribalski, B. S., Staveley-Smith, L., Kilborn, V. A., et al. 2004, AJ, 128, 16

Kourkchi, E., \& Tully, R. B. 2017, ApJ, 843, 16

Lee, J. C., Gil de Paz, A., Kennicutt, R. C., Jr., et al. 2011, ApJS, 192, 6
Lelli, F., Verheijen, M., \& Fraternali, F. 2014, MNRAS, 445, 1694 Leroy, A. K., Walter, F., Brinks, E., et al. 2008, AJ, 136, 2782 Leroy, A. K., Walter, F., Bigiel, F., et al. 2009, AJ, 137, 4670 Lucero, D. M., Carignan, C., Elson, E. C., et al. 2015, MNRAS, 450, 3935 Marasco, A., Fraternali, F., Heald, G., et al. 2019, A\&A, 631, A50 Mauch, T., Cotton, W. D., Condon, J. J., et al. 2020, ApJ, 888, 61 McMullin, J. P., Waters, B., Schiebel, D., Young, W., \& Golap, K. 2007, in Astronomical Data Analysis Software and Systems XVI, eds. R. A. Shaw, F. Hill, \& D. J. Bell, ASP Conf. Ser., 376, 127

Meurer, G. R., Hanish, D. J., Ferguson, H. C., et al. 2006, ApJS, 165, 307 Meurer, G. R., Wong, O. I., Kim, J. H., et al. 2009, ApJ, 695, 765 Moretti, A., Paladino, R., Poggianti, B. M., et al. 2020, ApJ, 897, L30 Mould, J. R., Huchra, J. P., Freedman, W. L., et al. 2000, ApJ, 529, 786 Muñoz-Mateos, J. C., Sheth, K., Regan, M., et al. 2015, ApJS, 219, 3 Murphy, E. J., Condon, J. J., Schinnerer, E., et al. 2011, ApJ, 737, 67 Nelson, D., Vogelsberger, M., Genel, S., et al. 2013, MNRAS, 429, 3353 Nelson, D., Genel, S., Vogelsberger, M., et al. 2015, MNRAS, 448, 59 Noterdaeme, P., Petitjean, P., Carithers, W. C., et al. 2012, A\&A, 547, L1 Pardy, S. A., D’Onghia, E., Athanassoula, E., Wilcots, E. M., \& Sheth, K. 2016, ApJ, 827, 149

Pilkington, K., Gibson, B. K., Calura, F., et al. 2011, MNRAS, 417, 2891 Popping, A., Davé, R., Braun, R., \& Oppenheimer, B. D. 2009, A\&A, 504, 15 Prieto, J. L., Lee, J. C., Drake, A. J., et al. 2012, ApJ, 745, 70

Putman, M. E., Peek, J. E. G., \& Joung, M. R. 2012, ARA\&A, 50, 491 Ramatsoku, M., Serra, P., Poggianti, B. M., et al. 2019, MNRAS, 487, 4580 Ramatsoku, M., Serra, P., Poggianti, B. M., et al. 2020, A\&A, 640, A22

Reynolds, T. N., Westmeier, T., Staveley-Smith, L., Chauhan, G., \& Lagos, C. D. P. 2020, MNRAS, 493, 5089

Richter, O. G., \& Sancisi, R. 1994, A\&A, 290, L9

Salpeter, E. E. 1955, ApJ, 121, 161

Sancisi, R., Fraternali, F., Oosterloo, T., \& van der Hulst, T. 2008, A\&ARv, 15, 189

Schaller, M., Dalla Vecchia, C., Schaye, J., et al. 2015, MNRAS, 454, 2277

Schmidt, M. 1959, ApJ, 129, 243

Sengupta, C., Scott, T. C., Verdes Montenegro, L., et al. 2012, A\&A, 546, A95

Serra, P., Westmeier, T., Giese, N., et al. 2015, MNRAS, 448, 1922

Serra, P., Maccagni, F. M., Kleiner, D., et al. 2019, A\&A, 628, A122

Solomon, P. M., \& Vanden Bout, P. A. 2005, ARA\&A, 43, 677

Sorgho, A., Carignan, C., Pisano, D. J., et al. 2019, MNRAS, 482, 1248

Thilker, D. A., Bianchi, L., Meurer, G., et al. 2007, ApJS, 173, 538

Tumlinson, J., Peeples, M. S., \& Werk, J. K. 2017, ARA\&A, 55, 389

van de Voort, F., Springel, V., Mandelker, N., van den Bosch, F. C., \& Pakmor, R. 2019, MNRAS, 482, L85

Venhola, A., Peletier, R., Laurikainen, E., et al. 2018, A\&A, 620, A165

Walter, F., Brinks, E., de Blok, W. J. G., et al. 2008, AJ, 136, 2563

Watts, A. B., Catinella, B., Cortese, L., \& Power, C. 2020, MNRAS, 492, 3672

Werk, J. K., Prochaska, J. X., Thom, C., et al. 2013, ApJS, 204, 17

Werk, J. K., Prochaska, J. X., Tumlinson, J., et al. 2014, ApJ, 792, 8

Westmeier, T., Braun, R., \& Koribalski, B. S. 2011, MNRAS, 410, 2217

Wolfe, S. A., Lockman, F. J., \& Pisano, D. J. 2016, ApJ, 816, 81

Young, J. S., \& Scoville, N. Z. 1991, ARA\&A, 29, 581

Zheng, Z., Meurer, G. R., Heckman, T. M., Thilker, D. A., \& Zwaan, M. A. 2013, MNRAS, 434, 3389

1 Netherlands Institute for Radio Astronomy (ASTRON), Oude Hoogeveensedijk 4, 7991 PD Dwingeloo, The Netherlands e-mail: blok@astron.nl

2 Dept. of Astronomy, Univ. of Cape Town, Private Bag X3, Rondebosch 7701, South Africa

3 Kapteyn Astronomical Institute, University of Groningen, PO Box 800, 9700 AV Groningen, The Netherlands

4 Aix Marseille Univ., CNRS, CNES, LAM, Marseille, France

5 Observatoire de Paris, Collège de France, Université PSL, Sorbonne Université, CNRS, LERMA, Paris, France

6 Department of Physics and Astronomy, University of Manitoba, Winnipeg R3T 2N2, Manitoba, Canada

7 CSIRO Astronomy and Space Science, PO Box 1130, Bentley, WA 6102, Australia

8 Ruhr-Universität Bochum, Faculty of Physics and Astronomy, Astronomical Institute, 44780 Bochum, Germany

9 Australia Telescope National Facility, CSIRO Astronomy and Space Science, PO Box 76, Epping, NSW 1710, Australia

10 Western Sydney University, Locked Bag 1797, Penrith South, NSW 1797, Australia 
11 ARC Centre of Excellence for All Sky Astrophysics in 3 Dimensions (ASTRO 3D), Australia

12 International Centre for Radio Astronomy Research, The University of Western Australia, 35 Stirling Highway, Crawley, WA 6009, Australia

13 Dep. to Astronomia Extragaláctica Istituto Astrofisica de Andalucía, Glorieta de la Astronomia s/n, 18008 Granada, Spain

14 Department of Astronomy, The Ohio State University, 140 West 18th Avenue, Columbus, OH 43210, USA

15 Center for Cosmology and Astroparticle Physics, 191 West Woodruff Avenue, Columbus, OH 43210, USA

16 Argelander-Institut für Astronomie, Auf dem Hügel 71, 53121 Bonn, Germany

17 Centre for Astrophysics Research, University of Hertfordshire, College Lane, Hatfield AL10 9AB, UK

18 Centro de Astronomía, Universidad de Antofagasta, Avda. U. de Antofagasta, 02800 Antofagasta, Chile

19 INAF - Osservatorio Astronomico di Cagliari, Via della Scienza 5, 09047 Selargius, CA, Italy

${ }^{20}$ Department of Physics \& Astronomy, West Virginia University, Morgantown, WV 26506, USA

21 Gravitational Waves and Cosmology Center, Chestnut Ridge Research Building, Morgantown, WV 26505, USA

22 Adjunct Astronomer, Green Bank Observatory, 155 Observatory Road, Green Bank, WV 24944, USA

23 Department of Physics and Space Science, Royal Military College of Canada, PO Box 17000, Station Forces, Kingston, Ontario K7K 7B4, Canada

${ }^{24}$ Laboratoire de Physique et de Chimie de l'Environnement, Observatoire d'Astrophysique de l'Université Ouaga I Pr. Joseph KiZerbo (ODAUO), 03 BP 7021, Ouaga 03, Burkina Faso
${ }^{25}$ E.A. Milne Centre for Astrophysics, University of Hull, Hull HU6 7RX, UK

26 Department of Physics and Astronomy, University of Louisville, 102 Natural Science Building, Louisville, KY 40292, USA

27 South African Radio Astronomy Oberservatory, Black River Park, 2 Fir Street, Observatory, Cape Town 7925, South Africa

28 Department of Physics and Electronics, Rhodes University, PO Box 94, Makhanda 6140, South Africa

29 Department of Physics, Virginia Polytechnic Institute and State University, 50 West Campus Drive, Blacksburg, VA 24061, USA

30 NASA Headquarters, 300 E Street SW, Washington, DC 20546, USA

31 Max Planck Institute for Astronomy, Königstuhl 17, 69117 Heidelberg, Germany

32 Jodrell Bank Centre for Astrophysics, School of Physics and Astronomy, University of Manchester, Oxford Road, Manchester M13 9PL, UK

33 Department of Astrophysics/IMAPP, Radboud University, PO Box 9010, 6500 GL Nijmegen, The Netherlands

34 Inter-University Institute for Data Intensive Astronomy \& Department of Astronomy, University of Cape Town, Private Bag X3, Rondebosch 7701, South Africa

35 South African Astronomical Observatory, PO Box 9, 7935 Observatory, South Africa

36 Leiden Observatory, Leiden University, PO Box 9513, 2300 RA Leiden, The Netherlands

37 IAU-Office For Astronomy for Development, PO Box 9, 7935 Observatory, South Africa

38 Center for Interdisciplinary Exploration and Research in Astrophysics (CIERA), Northwestern University, 1800 Sherman Ave., Evanston, IL 60201, USA 


\section{Appendix A: MHONGOOSE sample}

In Table A.1 we list some basic properties of the MHONGOOSE sample galaxies. A full description of the sample and the selection procedure will be the topic of a future paper.

Table A.1. MHONGOOSE sample.

\begin{tabular}{|c|c|c|c|c|c|c|c|}
\hline HIPASS & Name & $\begin{array}{l}\alpha(\mathrm{J} 2000.0) \\
(\mathrm{h} \mathrm{m} \mathrm{s}) \\
(3)\end{array}$ & $\begin{array}{l}\delta(\mathrm{J} 2000.0) \\
\left({ }^{\prime}, \prime \prime\right) \\
(4)\end{array}$ & $\begin{array}{r}D \\
(\mathrm{Mpc}) \\
(5) \\
\end{array}$ & $\begin{array}{r}V_{\text {hel }} \\
\left(\mathrm{km} \mathrm{s}^{-1}\right) \\
(6)\end{array}$ & $\log \left(\frac{M_{\mathrm{HI}}}{M_{\odot}}\right)$ & $\begin{array}{r}W_{50} \\
\left(\mathrm{~km} \mathrm{~s}^{-1}\right) \\
(8)\end{array}$ \\
\hline \multicolumn{8}{|c|}{ Bin: $6.0<\log \left(M_{\mathrm{HI}} / M_{\odot}\right)<8.0$} \\
\hline J0008-34 & ESO 349-G031 & 000813.36 & -343442.0 & 3.3 & 221.0 & 7.17 & 30 \\
\hline J0049-20 & UGC A015 & 004949.20 & -210054.0 & 3.3 & 294.6 & 6.99 & 36 \\
\hline J0310-39 & ESO 300-G016 & 031010.48 & -400010.5 & 9.3 & 709.7 & 7.95 & 36 \\
\hline J0454-53 & NGC 1705 & 045413.50 & -532139.8 & 5.1 & 633.3 & 7.96 & 128 \\
\hline $\mathrm{J} 1321-31$ & KK 98-195 & 132108.20 & -313145.0 & 5.2 & 571.8 & 7.56 & 38 \\
\hline \multicolumn{8}{|c|}{ Bin: $8.0<\log \left(M_{\mathrm{HI}} / M_{\odot}\right)<8.5$} \\
\hline J0031-22 & ESO 473-G024 & 003122.51 & -224557.5 & 7.9 & 539.2 & 8.01 & 47 \\
\hline J0135-41 & NGC 0625 & 013504.63 & -412610.3 & 4.1 & 395.7 & 8.09 & 75 \\
\hline J0320-52 & NGC 1311 & 032006.96 & -521107.9 & 7.0 & 569.1 & 8.25 & 81 \\
\hline J0429-27 & NGC 1592 & 042940.13 & -272430.7 & 13.0 & 946.0 & 8.37 & 73 \\
\hline $\mathrm{J} 1337-28$ & ESO 444-G084 & 133719.99 & -280242.0 & 4.6 & 587.0 & 8.03 & 58 \\
\hline \multicolumn{8}{|c|}{ Bin: $8.5<\log \left(M_{\mathrm{HI}} / M_{\odot}\right)<9.0$} \\
\hline J0331-51 & IC 1954 & 033131.39 & -515417.4 & 14.3 & 1063.4 & 8.96 & 224 \\
\hline J0351-38 & ESO 302-G014 & 035140.90 & -382708.0 & 11.7 & 871.7 & 8.55 & 67 \\
\hline J1106-14 & KKS 2000-23 & 110612.00 & -142425.7 & 12.7 & 1039.3 & 8.62 & 77 \\
\hline $\mathrm{J} 1253-12$ & UGC A307 & 125357.29 & -120621.0 & 8.6 & 824.2 & 8.67 & 72 \\
\hline J2009-61 & IC 4951 & 200931.77 & -615101.7 & 11.3 & 814.4 & 8.87 & 122 \\
\hline \multicolumn{8}{|c|}{ Bin: $9.0<\log \left(M_{\mathrm{HI}} / M_{\odot}\right)<9.5$} \\
\hline J0309-41 & ESO 300-G014 & 030937.87 & -410149.7 & 12.9 & 955.0 & 9.00 & 130 \\
\hline J0546-52 & NGC 2101 & 054624.17 & -520518.7 & 16.1 & 1192.3 & 9.18 & 93 \\
\hline $\mathrm{J} 1303-17 \mathrm{~b}$ & UGC A320 & 130316.74 & -172522.9 & 7.7 & 742.9 & 9.12 & 113 \\
\hline J1318-21 & NGC 5068 & 131854.81 & -210220.8 & 6.9 & 668.1 & 9.16 & 73 \\
\hline $\mathrm{J} 2357-32$ & NGC 7793 & 235749.83 & -323527.7 & 3.9 & 227.3 & 9.01 & 173 \\
\hline \multicolumn{8}{|c|}{ Bin: $9.5<\log \left(M_{\mathrm{HI}} / M_{\odot}\right)<10.0$} \\
\hline J0335-24 & NGC 1371 & 033501.34 & -245559.6 & 20.4 & 1462.9 & 9.84 & 391 \\
\hline J0459-26 & NGC 1744 & 045957.80 & -260120.0 & 10.0 & 740.8 & 9.56 & 200 \\
\hline J0516-37 & ESO 362-G011 & 051638.80 & -370609.1 & 18.7 & 1344.0 & 9.68 & 280 \\
\hline $\mathrm{J} 1103-23^{(a)}$ & NGC 3511 & 110323.77 & -230512.4 & 14.2 & 1113.9 & 9.62 & 265 \\
\hline $\mathrm{J} 1254-10 \mathrm{a}$ & NGC 4781 & 125427.00 & -103030.0 & 16.1 & 1260.1 & 9.55 & 233 \\
\hline \multicolumn{8}{|c|}{ Bin: $10.0<\log \left(M_{\mathrm{HI}} / M_{\odot}\right)<10.5$} \\
\hline J0052-31 & NGC 0289 & 005242.36 & -311221.0 & 22.9 & 1628.9 & 10.34 & 277 \\
\hline J0419-54 & NGC 1566 & 042000.42 & -545616.1 & 20.7 & 1503.0 & 10.19 & 205 \\
\hline J0445-59 & NGC 1672 & 044542.50 & -591449.9 & 18.1 & 1334.0 & 10.19 & 250 \\
\hline J1153-28 & UGC A250 & 115324.06 & -283311.4 & 24.4 & 1706.5 & 10.00 & 274 \\
\hline J2257-41 & NGC 7424 & 225718.37 & -410414.1 & 13.6 & 938.5 & 10.04 & 159 \\
\hline
\end{tabular}

Notes. ${ }^{(a)}$ Pair with NGC 3513. Columns: (1) HIPASS identification. (2) Other name. (3) Right ascension (J2000.0). (4) Declination (J2000.0). (5) Distance (Mpc). (6) Heliocentric velocity $\left(\mathrm{km} \mathrm{s}^{-1}\right.$ ). (7) Logarithmic H I mass. (8) W5 H I velocity width. Galaxy properties from Meurer et al. (2006). 